T. De Martino - M. Fontana - F. Giannini - M. Meirana

\title{
A Free Form Feature Taxonomy
}

Rapporto Tecnico IMA $-\mathbf{n . 8 / 9 8}$ 


\section{CONTENTS}

\section{Introduction}

2 Form features for aesthetic design .................................3

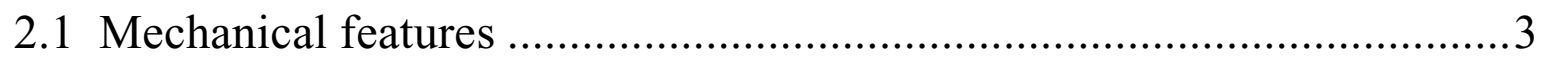

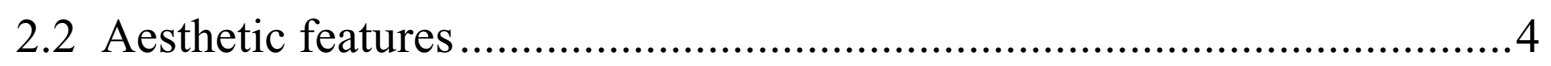

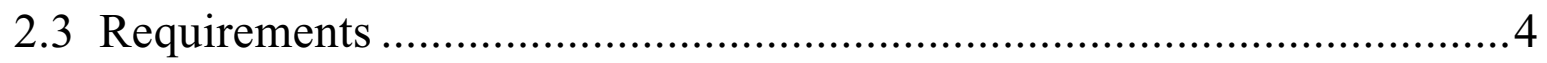

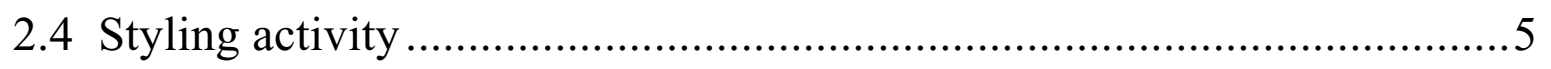

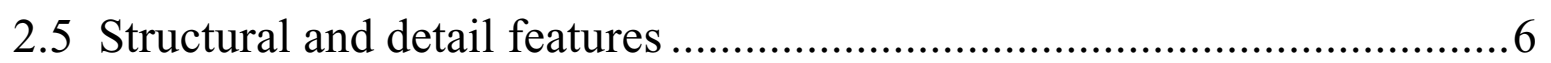

\section{Detail features ................................................................}

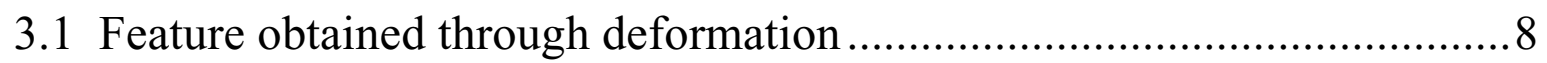

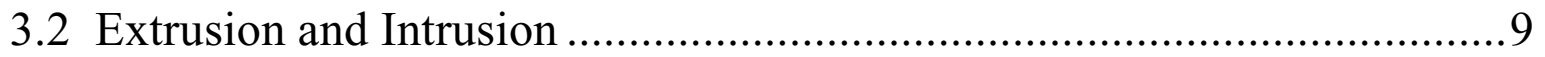

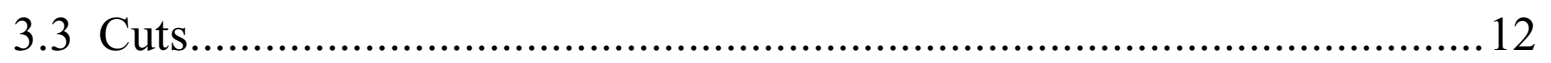

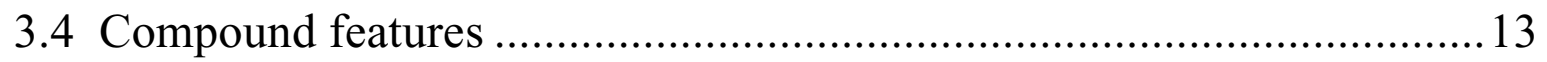

4 Types of simple features .................................................13

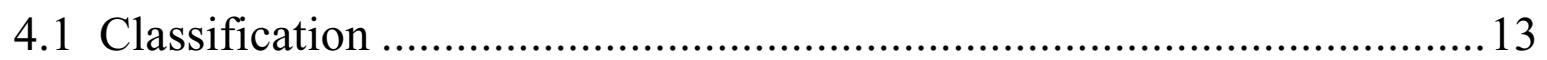

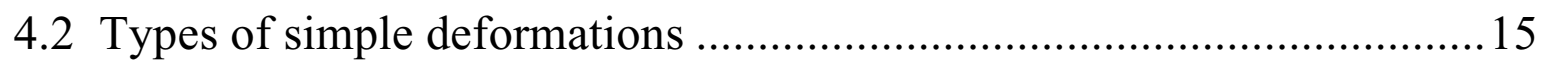

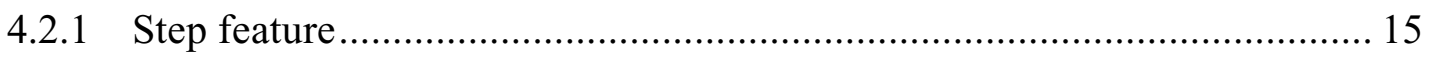

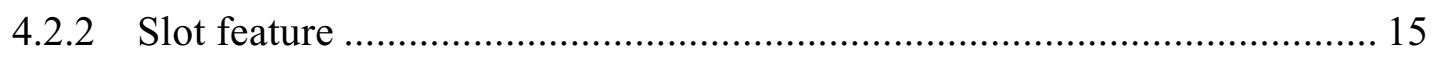

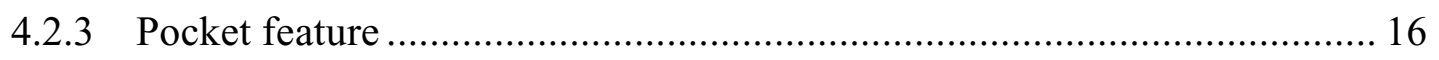

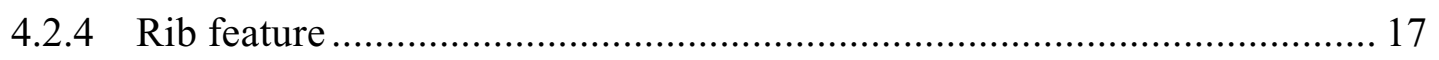

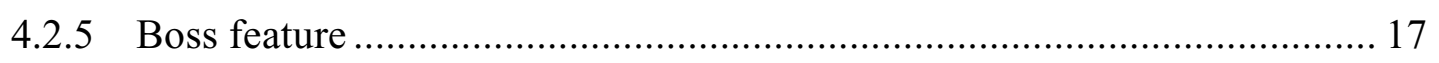

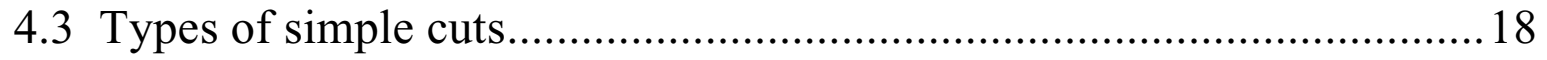

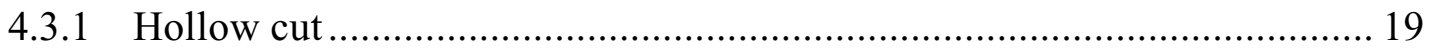

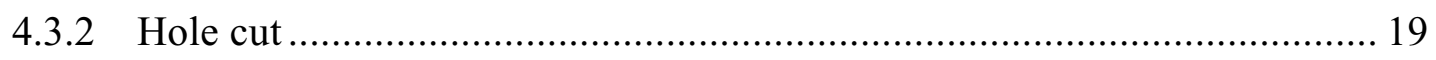

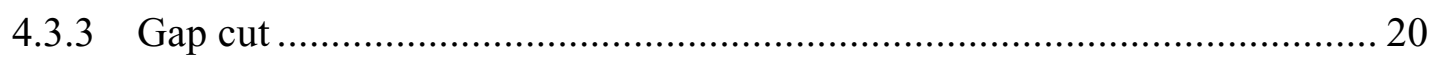




\section{Definition of geometric parameters...............................21}

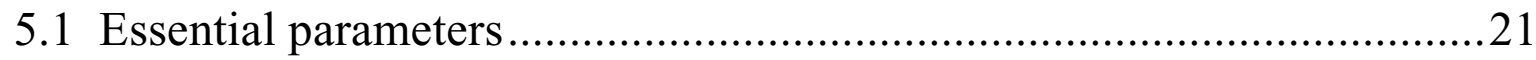

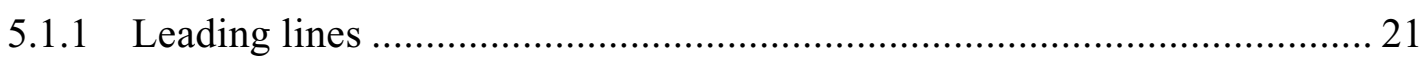

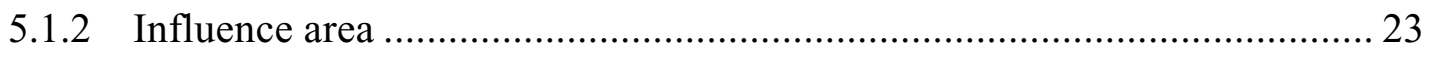

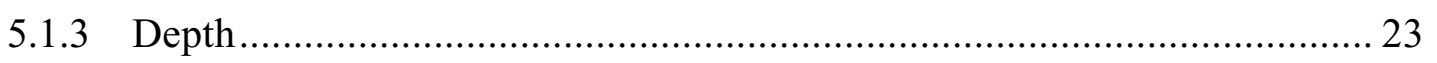

5.1.4 Transition conditions ........................................................................... 24

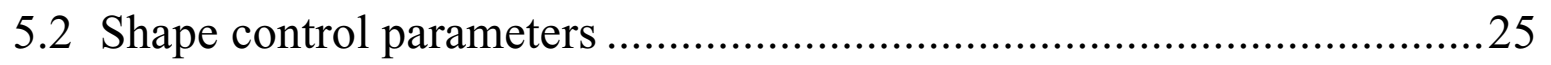

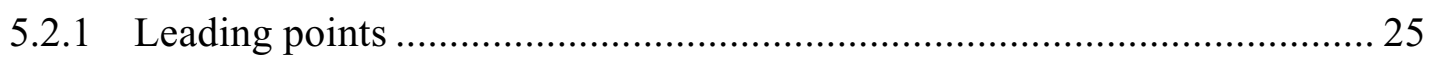

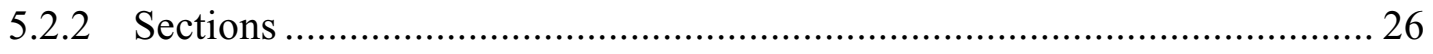

5.2.3 Curvature and gradient laws................................................................. 28

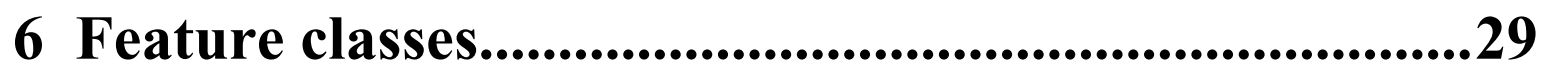

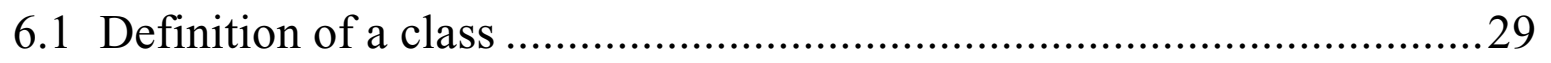

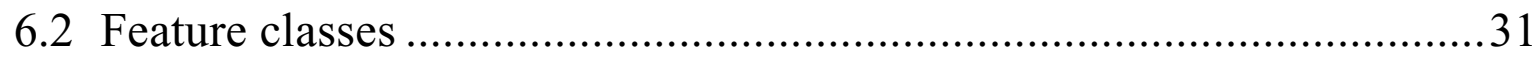

Acknowledgements ..................................................................43

Bibliography.............................................................................43 


\section{Introduction}

In this document the notion of form feature for aesthetic design is presented. The design of industrial products constituted by free-form surfaces is done by using CAD systems representing curves and surfaces by means of NURBS functions, which are defined by low level entities that are not intuitive and require some knowledge of the mathematical language. Similarly to the featurebased approach adopted by CAD systems for classical mechanical design, we identify a set of high level modelling entities which provides commonly performed shape modifications.

Particularly, the so-called detail features are examined for an aesthetic and/or functional characterization of predefined free-form surfaces and a classification is suggested, considering a set of possible intuitive parameters (geometric entities and relations among them). Any modification of form features will be then possible by varying values for such parameters, according to the designer's requirements for aesthetic purposes.

The paper is organized as follows.

Chapter 2 introduces the feature concept for aesthetic design then differences among mechanical form features and aesthetic features are shown, and two types of features for aesthetic design are distinguished: structural features and detail features. Fundamental properties of the latter are then described.

In chapter 3 a general classification of detail features is proposed: it is based on the types of deformation and elimination produced over a surface.

In chapter 4 specific definitions for elementary feature types and some examples are shown.

Chapter 5 describes the geometric parameters used for the definition and the shape control of a detail feature aimed to an object oriented classification of feature types.

The formal definition of each feature class will be finally described in chapter 6 according to the inheritance approach of the object oriented technology in terms of the father class, the name, the essential and shape control parameters and the constraints on parameters to be respected.

\section{Form features for aesthetic design}

\subsection{Mechanical features}

Form features have been introduced in mechanical environments as the key elements for associating specific functional meaning to groups of geometric elements (faces, edges and vertices), thus offering the advantage of treating sets of elements as unique entities [1]. Such entities are much more meaningful for application than the low level constitutive elements and are manipulated by means of a limited number of significant parameters. It is quite clear that using features as design primitives improves the efficiency in creating the product model, in considering solution alternatives and shortens the time required for model changes.

In order to define a feature-based design system, it is necessary to identify the set of primitives (features) that are useful for the selected application domain. As previously mentioned, the concept of feature has been mainly investigated in mechanical environments, this is mainly due to the fact that classical mechanical parts are defined by canonical geometric shapes, which can be easily classified, and the association between shape and function can be better identified:

In Fig. 1 we report some types of form features used in mechanical design. 


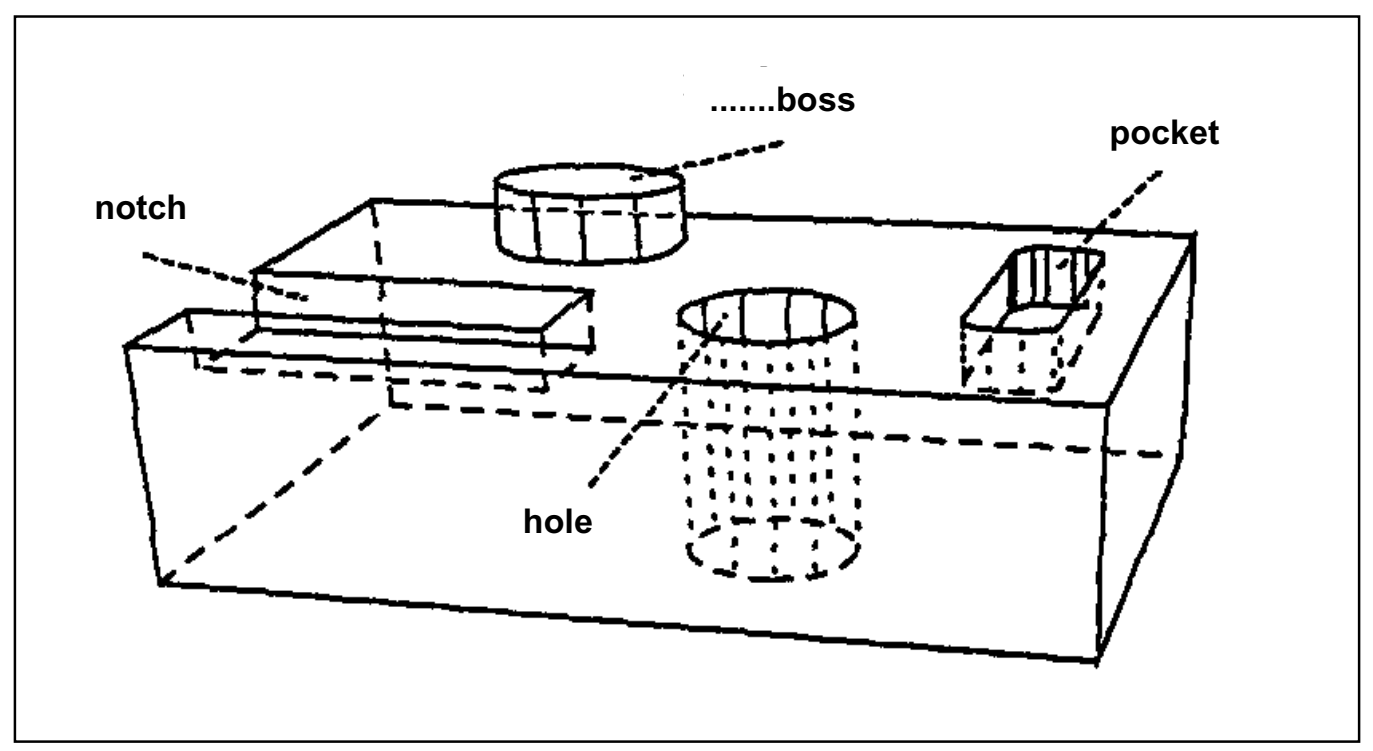

Fig. 1: Mechanical form features

\subsection{Aesthetic features}

Trying to define a concept of form feature which can be used in activities for aesthetic design, we intend to represent parts of an object having an aesthetic meaning, instead of a function.

As regards this, not all the types of features defined for mechanical CAD systems can be directly used in the frame of aesthetic design. In this case the designer needs a certain creativity, a certain freedom of expression that takes into account personal tastes, visual impressions, fashions, etc. which cannot be achieved by means of the rigid shapes constituting mechanical features. What can be recovered is in any case a methodology in the modelling activity: features as complex high level entities which allow a fast creation and modification of the geometric model.

In both contexts (mechanical and aesthetic design) what makes one feature different from another are the geometric and topological elements which define the shape and the appearance of the feature, and also the type of interaction with the remaining geometry of the object. A form feature is what make 'different' (functionally in the mechanical design, and aesthetically in the styling activities) two objects which were initially built by the same overall geometry (for example, the same surface or the same shape as support).

\subsection{Requirements}

In order to provide significant modelling entities, an aesthetic feature has to satisfy the following basic requirements:

- it is a part constituting the geometry of the product;

- it has geometric and topological properties predictable in a univocal way, so that it is reproducible;

- it gives a peculiar aesthetic impression and characterization to the product where it is positioned. 


\subsection{Styling activity}

In order to identify the set of features meaningful for aesthetic design, the working methods of stylists have been analysed. The considered questions are: what are the common ideas followed by the designers when they sketch a shape? What are the criteria used?

Observing how designers define the aspect of the products, it appears that the definition of a shape is performed in two logical steps:

- Overall shape definition, providing the product global effect;

- Detail definition, originating the complete final shape of the product.

This two steps approach is used both for hand sketching and for CAS/CAD modelling. In particular, in the computer assisted activity, in the first phase the designer creates the object's enveloping surfaces from some essential curves that bound the overall shape. These curves can be thought as the skeleton of the product model, that is what would be drawn by hand for visualizing the object, and correspond not only to the object profiles and selected sections, but also to significant lines strongly affecting the product shape, i.e. the so-called character lines $[8,1]$. The quality and the aesthetics of such curves is quite important since they are used for creating the surfaces enveloping the product, thus the global product impression is strongly dependent on their shape. Thus, many checks are made on them, with different tools, since their modification when the product model is almost complete is very cumbersome requiring the manual modification of most of the created surfaces.

In Fig. 2, an example of quality analysis of the structural curves: the horizontal compression is for emphasising the curvature defects.

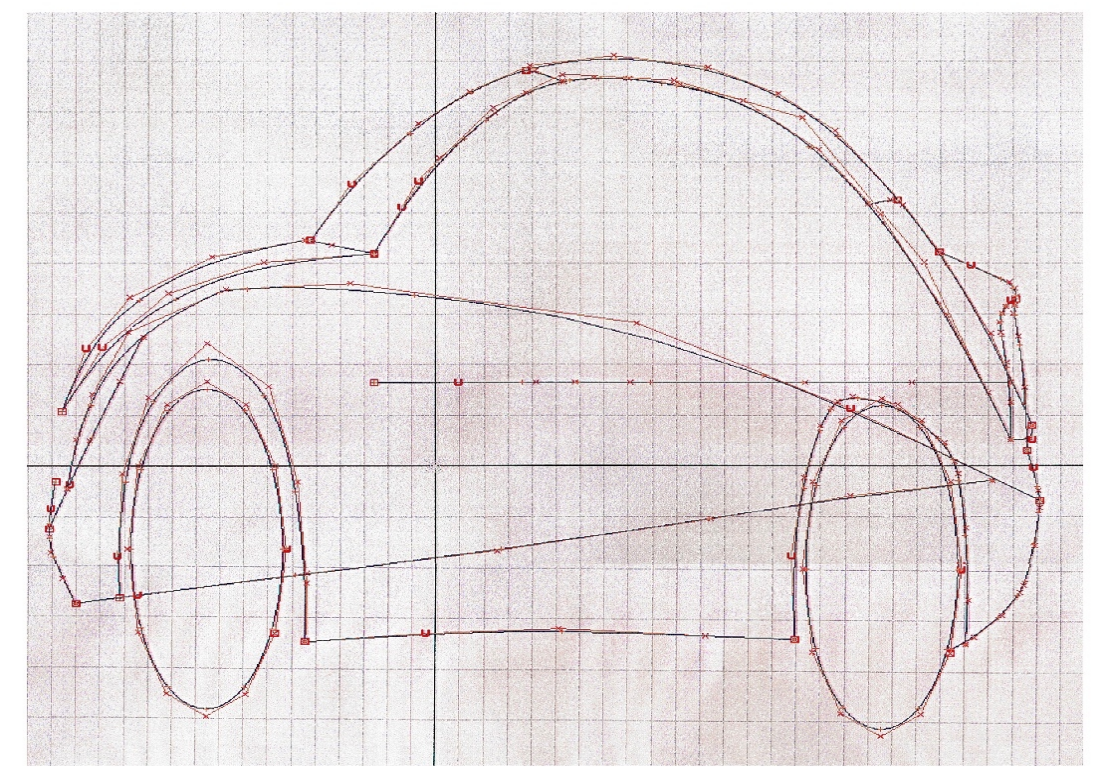

Fig. 2: Compressed structural curves of a car model (by PininFarina)

In the second step, the details that characterize the object functionally and aesthetically are added. This correspond to a modification of the surfaces previously created possibly with the creation of new surfaces.

At this point is important to notice that while specific contours can be really created as curves when creating the surface based model of the product, most of the character lines are virtual (i.e. only perceived) lines, thus they are as a matter of fact obtained as set of surfaces or through particular 
relations among surfaces (see Fig. 3). Thus they are created in the second design phase. Figure 3 shows examples of these kinds of character lines.

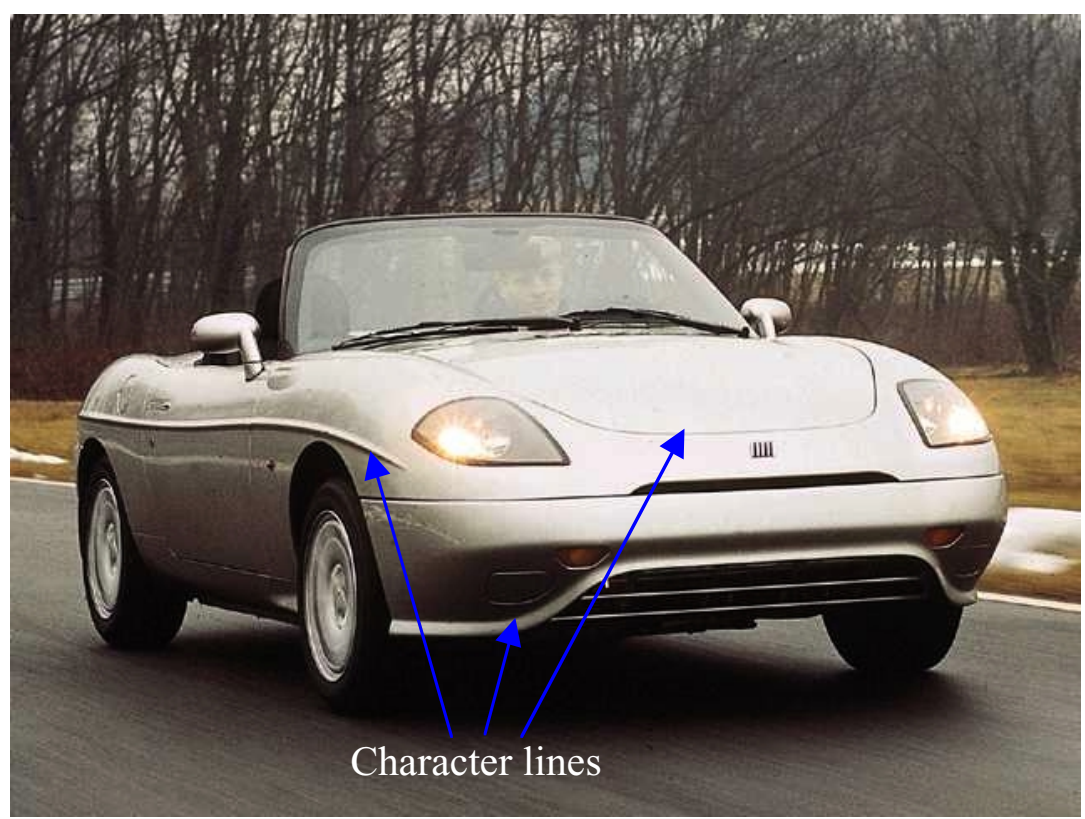

Fig. 3: Barchetta (by Fiat)

\subsection{Structural and detail features}

According to the different phases of the design activity in styling, and keeping in mind the requirements that a feature has to satisfy, it is possible to identify two categories of form features in the aesthetic design:

1. Structural features, created in the preliminary phase of design. They are the structural entities used for defining the surfaces constituting the product, thus having an important aesthetic impact.

2. Detail features, created in the second modelling phase. They are applied on a surface for adding aesthetic and functional details and for enforcing the visual effects of character lines.

Features of the first kind serve to group connected set of curves according to their intrinsic meaning (i.e. contours and character lines) to treat them as a unique entity and to maintain the relationships with the corresponding derived surfaces. Thus, constituting the basis for an easier and more efficient successive modification of the whole product by varying the shapes of the structural elements.

Examples of structural features are shown in Fig. 4, where some lines (contours and character lines), which characterize the shape aspect of the car body of the Nautilus designed by Pininfarina, are highlighted. 


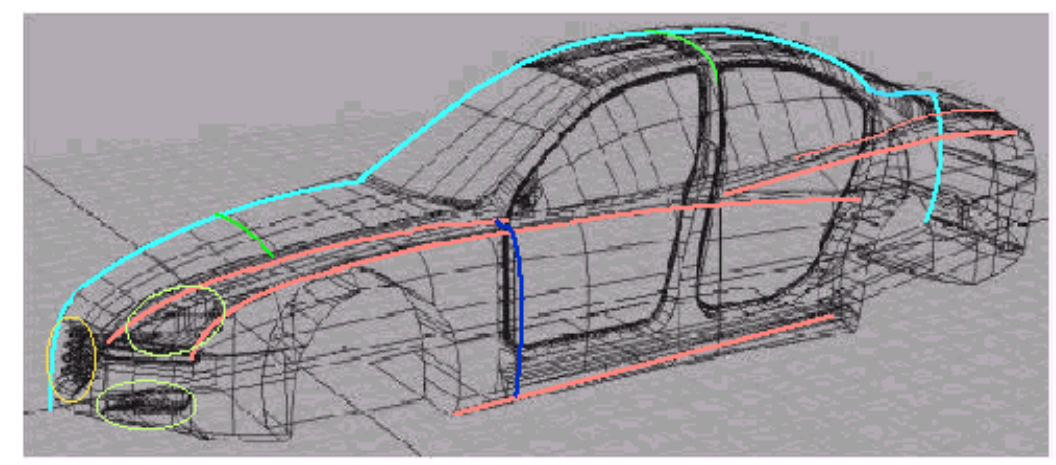

Fig. 4: Nautilus (Pininfarina)

The current document focuses on detail features, and in particular, it will treat mainly features aimed at enforcing the visual effect of virtual curves.

Features of the second type are used in the a posteriori modelling approach: starting from a freeform surface in which features are generated for characterizing and completing the specification of the product. Since we consider form features, we can identify them as local modifications on a primary surface, consisting in more or less marked variations that make in a mathematical and aesthetic sense the resulting surface somehow 'different' from the former. They correspond to a group of adjacent more or less extended surfaces and most of the cases, they are associated to a character line.

Since a detail feature can be seen as a local modification of a surface, from the mathematical point of view we have a local redefinition of the surface description and also a possible (but not necessary) local variation of the degree of continuity with respect to the original surface. Regarding this, as an example we can think of detail features with breaks of parts by means of cuts and/or holes: that implies that a continuity condition has been lost (see chapter 5.1.4 about geometric continuity). This mathematical level can suggest another approach: the feature can be seen as an operator which associates to the original surface a new surface transformed by the latter $[11,12$, 13].

\section{Detail features}

The purpose of this and the following sections is to provide a set of simple features, corresponding to local modifications of given free-form surfaces, sufficient to create object having complex shapes. These features have to cover in a sufficiently exhaustive way the three possible operations that can be done on a local part of a surface:

- adding a region,

- substituting a region;

- removing a region.

While the first operation may be performed by union operations on already defined shapes, or through the use of modelling operations such as fillets and blendings that can be considered also as features but are not treated here since already well known, the latter two operations can be associated to the two-fold idea of deformation and elimination. In the following section the two concepts are described. 


\subsection{Feature obtained through deformation}

Both in case of a deformation (substitution of parts) and an elimination (removal of parts), given a free-form surface, a new surface is generated from the former. The difference is in that the operation of deformation transforms points into points, while in the operation of elimination points are removed.

We define then as deformation law the transformation criterion that is applied to a surface for obtaining the modified surface corresponding to the detail form feature. The deformation law might be implemented as a sequence of predefined modelling operations or as a mapping function that transforms points of the primary surface into points of the modified surface, (only in case of deformation features). In the case of features corresponding to the removal of a surface region the deformation law might simply correspond to a trim operation. The set of removed points has to form a dense set, i.e. points are not isolated (practically a portion of surface is removed). See on this regard Fig. 5.

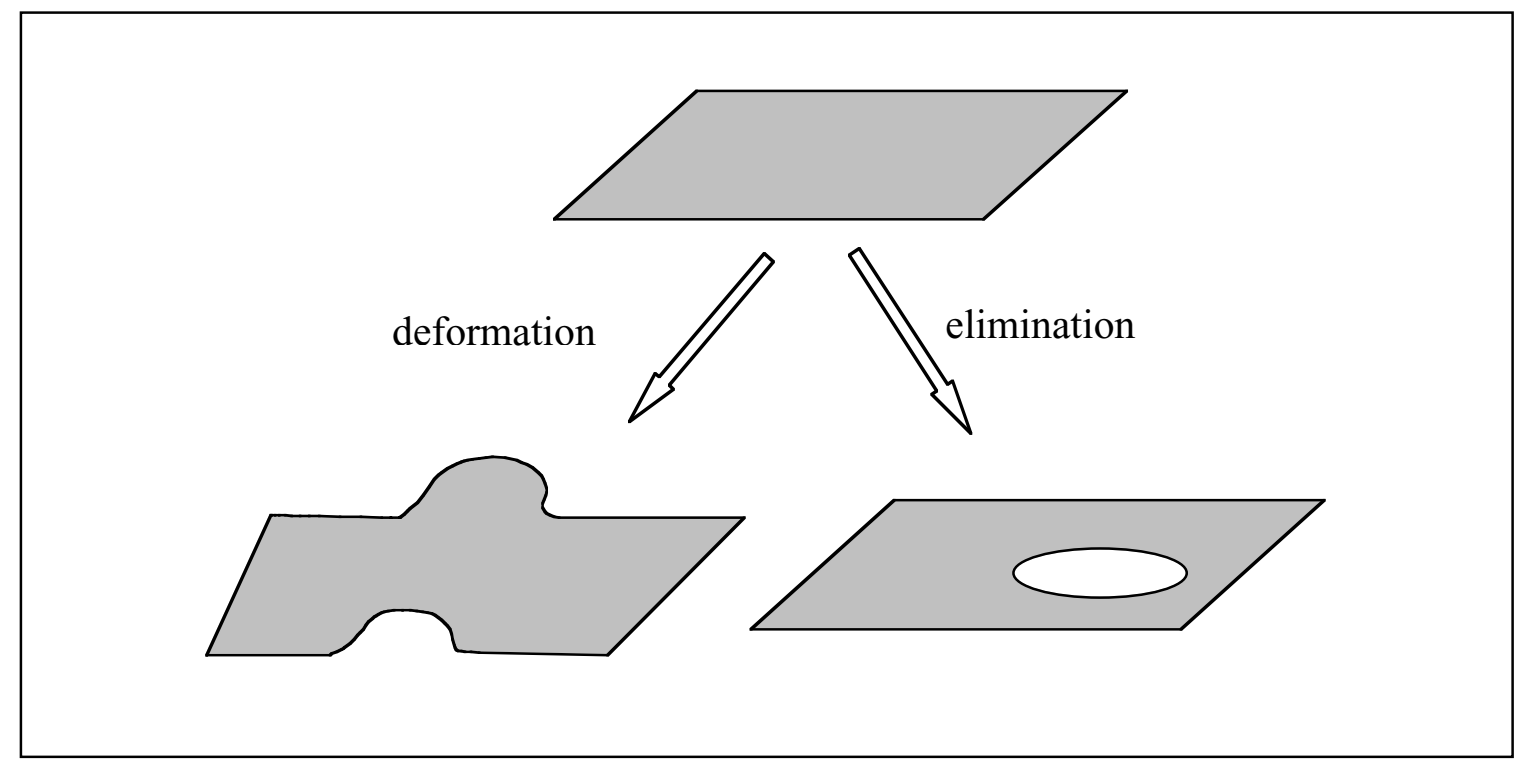

Fig. 5: Deformation and elimination operations applied on a surface

In both case, we will indicate as primary surface the patch, or a set of adjacent patches, to be modified by a detail feature and characterized surface the resulting transformed surface.

Considering a feature as a surface deformation, possibly an elimination, it is important to define some key elements:

- the area of the surface that has to be modified;

- the type of deformation to be performed;

- which is the behaviour along the contacts among the modified and the unmodified parts.

In order to specify the classes of deformation which are possible, it is necessary to provide some definition and work hypothesis.

Regarding the first point, we call influence area of the feature the part of the primary surface which has to be modified by the insertion of the feature, and influence boundary the corresponding border. For easier definition purposes we will consider the hypothesis that all the surfaces composing the primary surface have a coherent normal directions, i.e. all normal unit vectors to the surface point towards the the object exterior. See in Fig. 6 an example of coherent surfaces. This is not a too 
restrictive hypothesis since it is always possible to choose a parametrization for the surfaces such that the above condition is satisfied.

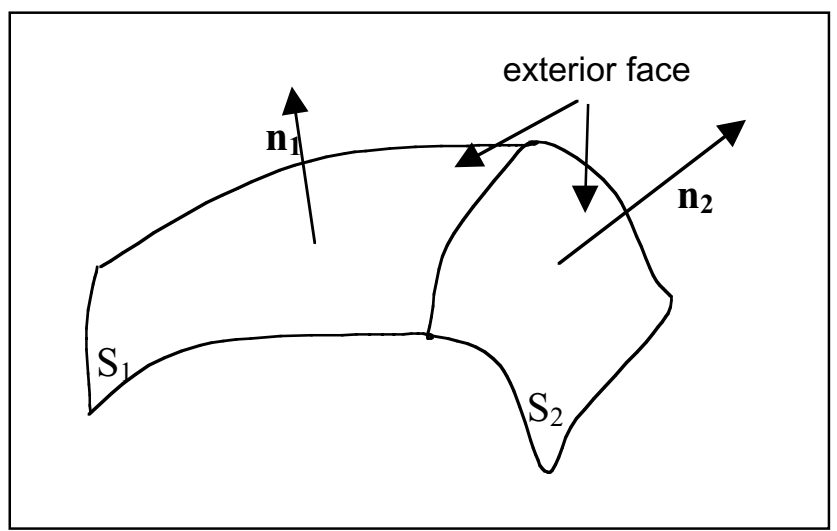

Fig. 6: Coherent normal direction of adjacent patches

Besides, it is reminded that:

Given two vectors $\mathbf{u}$ and $\mathbf{v}$ in $R^{3}$, it is said that $\mathbf{u}$ is concordant with $\mathbf{v}$ if their scalar product $\mathbf{u} \% \mathbf{v}$ is positive and discordant if it is negative

The idea of deformation and elimination is then associated to the following three feature categories (see the scheme in Fig. 7):

1. extrusions,

2. intrusions,

3. cuts.

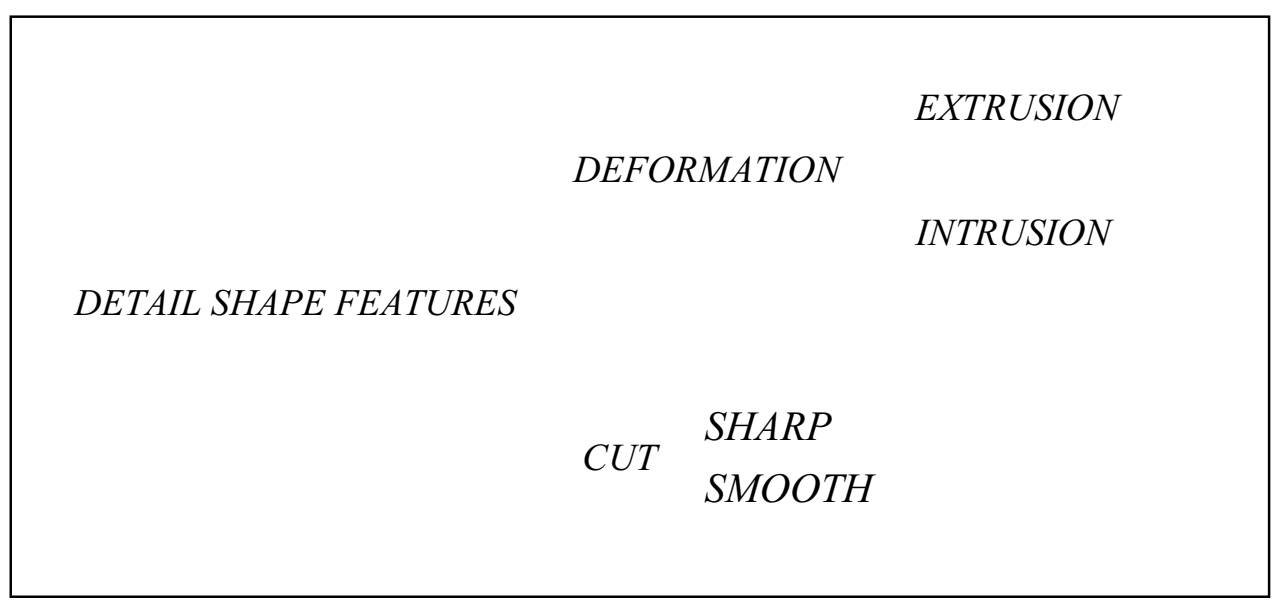

Fig. 7: General classification of detail features

\subsection{Extrusions and Intrusions}

The concepts of extrusion and intrusion on a surface have to represent the ideas of a local deformation 'upwards' (direction concordant with the surface normal unit vector) and 'downwards' (discordant) respectively, and both do not imply any removal of parts, differently from the concept of cut.

For a more formal feature types description, we introduce the following definition: 
Given a primary surface $S$ and the influence area $A_{I} \mid S$, a deformation law is a function $\delta: S \diamond R^{3}$, where $\delta\left(A_{I}\right)=S^{\prime}$ is the characterized surface, such that $\delta$ is the identity function in $S-A_{I}$.

Therefore (see Fig. 8), given the deformation law $\delta$ :

1. An extrusion is the characterized surface $S$ ' having the following property: for each point $P\left\lfloor A_{I} \mid S\right.$, considered the corresponding $P^{\prime}=\delta(P)$ the oriented vector $\overrightarrow{P P}^{\prime}$ is concordant with the normal unit vector $\mathbf{n}$ at the surface $S$ in $P$.

2. An intrusion is the characterized surface $S$ ' having the following property: for each point $P\left\lfloor A_{I}\right.$, considered $P^{\prime}=\delta(P)$ the oriented vector $\overrightarrow{P P^{\prime}}$ is discordant with the normal unit vector n at the surface $S$ in $P$

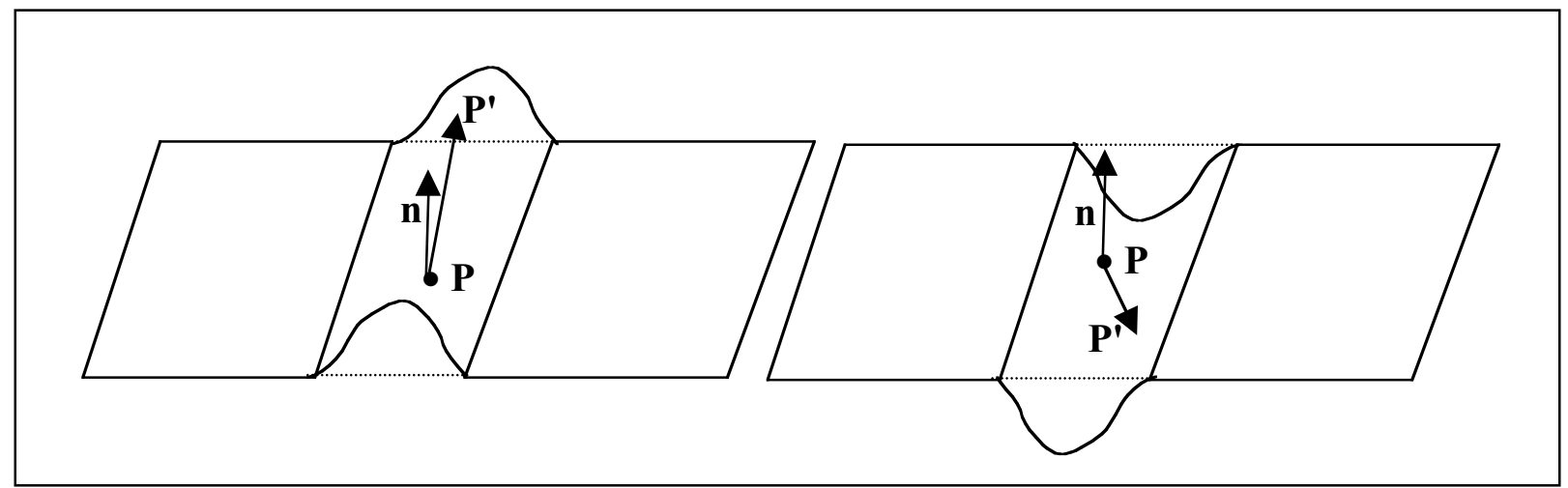

Fig. 8: Examples of extrusions and intrusions

As previously mentioned S may indicate a unique or a set of adjacent patches, consequently $A_{I}$ and $S$ ' itself may be a portion of a unique or of several adjacent patches.

The general definition given for extrusions and intrusions allows to introduce both deformations without break and deformations with break. In the first case, the continuity condition along the influence boundary is respected, at least the so-called $G^{0}$ condition (see chapter 5), and the resulting characterized surface has to maintain the same connection degree of the original primary surface. Intuitively we have the idea of a deformation without any jump. On the contrary, in the second case, there is discontinuity along a portion of the influence boundary, $i$. e. there is at least a segment along which there is geometric continuity $G^{-1}$, and the characterized surface results in any case non simply connected. Here we associate the idea of 'jump'.

A detail feature, representing a type of deformation with break, is shown in Fig. 9. 


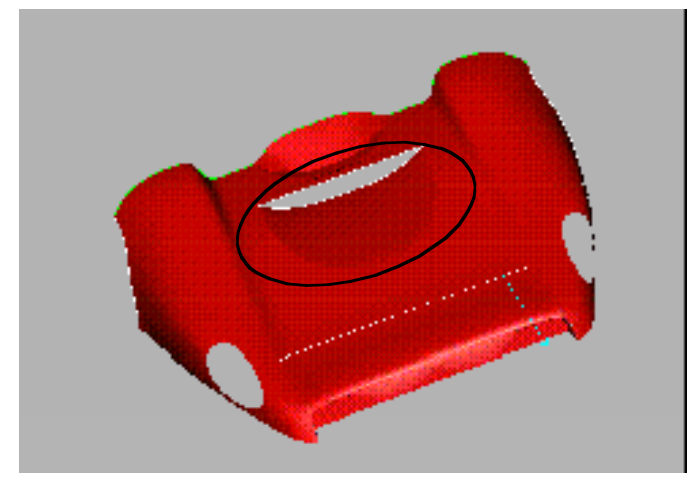

Fig. 9: Example of deformation with break

It is possible to point out a particular category of detail features [6] by deformation which are obtained by means of a rigid translation of parts, precisely:

A displacement feature is defined as a particular intrusion or extrusion in which a part is obtained through a pure rigid translation of a part of the primary surface inside the influence area along a constant direction non tangent at the surface.

See Fig. 10 as an example of displacement type obtained by raising a planar surface.

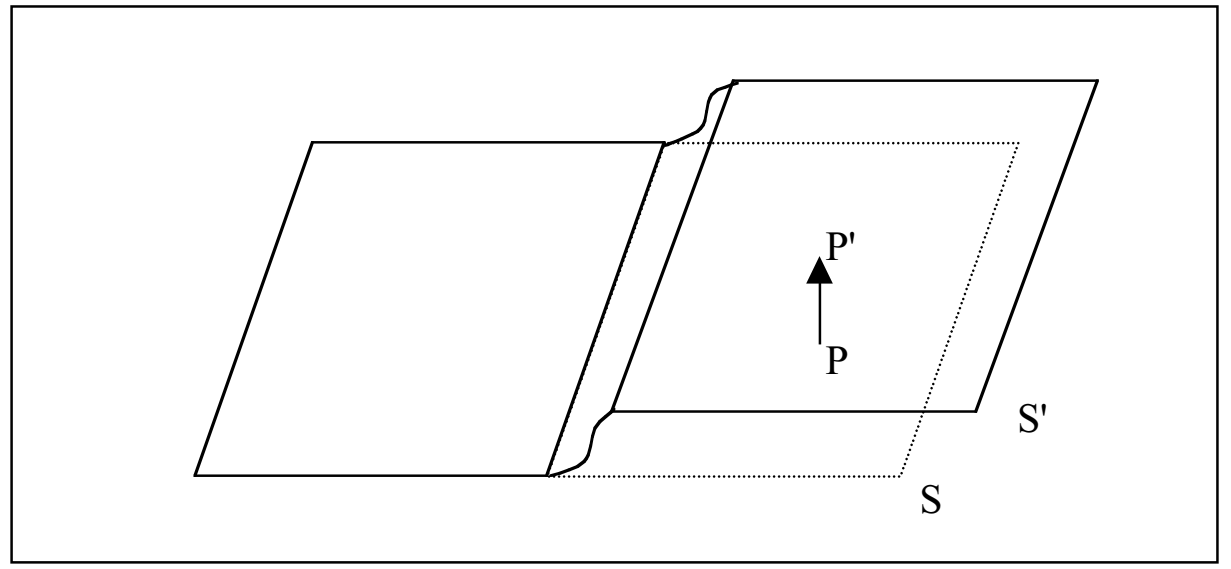

Fig. 10: Representation of an extrusion by displacement

If $S$ and $S^{\prime}$ are respectively the primary surface and the corresponding characterized surface obtained by means of an intrusion or an extrusion with displacement, then a region $A_{D} \mid A_{I}$ (called displacement area) exists such that $\forall P\left\lfloor A_{D}\right.$ the transformed point $P^{\prime}$ in $S^{\prime}$ is obtained as $P^{\prime}=P+h \mathbf{v}$, where $\mathbf{v}$ is the constant unit vector (we can in any case assume it as concordant with the surface normal unit vector) representing the offset direction and $h$ is a fixed constant scalar, positive or negative depending whether we have an extrusion or intrusion type, respectively.

In the area where the deformation does not cause breaks, displacement features are supported by a transition surface which blends the unmodified region with the displaced part. Generally variations of curvature between the blending surface and the displacement make a character line well singled out, separating the two parts.

It could also be possible to consider intrusions and extrusions created through more general rigid motions consisting in simultaneous rotations and translations of parts of the primary surface, matched with transition surfaces for the blending between the displaced and the unchanged part. 


\subsection{Cuts}

Now it is possible to define an elimination of a surface region as follows:

$A$ cut is a modification of a primary surface which gives raise to a surface $S^{\prime}=S-S_{T}$, where $S_{T}$ is a simply connected surface whose support is contained in $S$.

In the following chapter we will describe several types of elementary cuts according to properties of connection characterizing the removed surface.

In Fig. 11 we report several types of cuts that can be made on a surface.

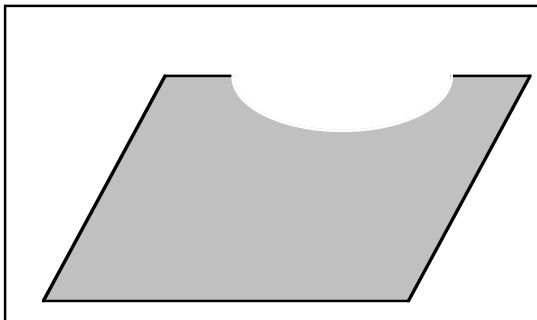

(a)

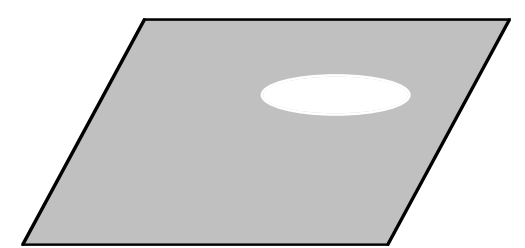

(b)
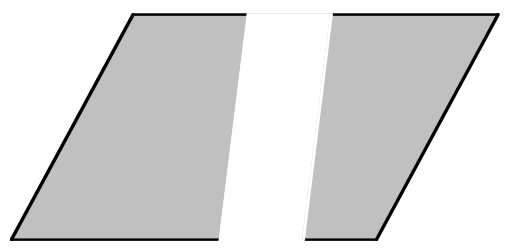

(c)

Fig. 11: Examples of cuts

For aesthetic purposes, the surface along the profile of the cut may need to be smoothed. This can be done, for example, by adding a fillet and a refining surface (see Fig. 12). Let us think of a soft lifting up/sinking made on the edges of the non removed region (for instance, the part of a car body surrounding the wheel from above). Therefore it is possible to distinguish two types of cut:

- sharp cut, when no rounding conditions are specified,

- smooth cut, when refining surfaces are present.

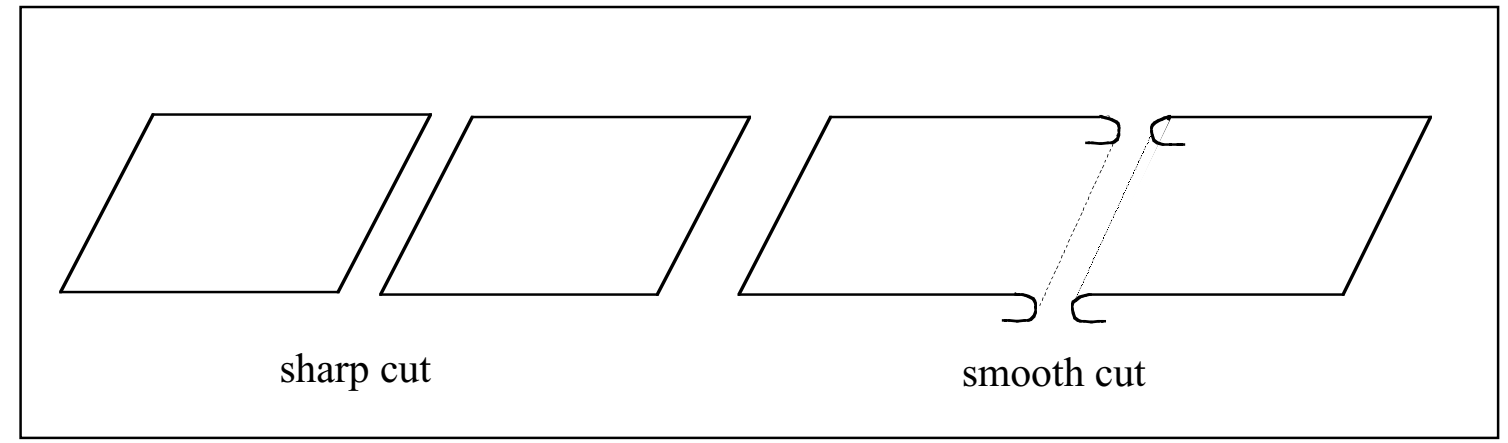

Fig. 12: Derivation of a smooth cut from a sharp cut

The sharp cut requires only trimming operations. The identification of rules for setting the refinement conditions for cuts requires a deeper analysis in which aesthetic criteria have to be considered together with the optical effects due to particular bendings of the surface and feasibility restrictions depending on the material and the production technologies. 


\subsection{Compound features}

In the previous paragraph simple detail form features have been considered. However it is possible to define other features which can be obtained by structured compositions of simple features, thus using results of complex modelling operations.

More precisely, the user can define a compound detail feature as the result of the generation of a certain number of successive simple feature (intrusions, extrusions and cuts).

A particular type of compound features are the pattern-features. They are defined as shapes identifiable as a unique entity which are obtained by several feature instances bound together by prearranged relations. Let us think of distributions of simple features like sequences of cavities on a surface having the same shape and placed according to a fixed scheme $[4,5]$.

The definition of a compound feature does not require that the component features are sufficiently 'near': the influence areas of the corresponding constituting features can be disjoint or not. In any case, evidently, the compound feature has as resulting influence area the union of the influence areas of the single component features.

In Fig. 13 some examples of compound detail features are given, and in Fig. 14 a pattern-feature given by a sequence of cuts made over arch-shaped intrusions with a similar behaviour is depicted.

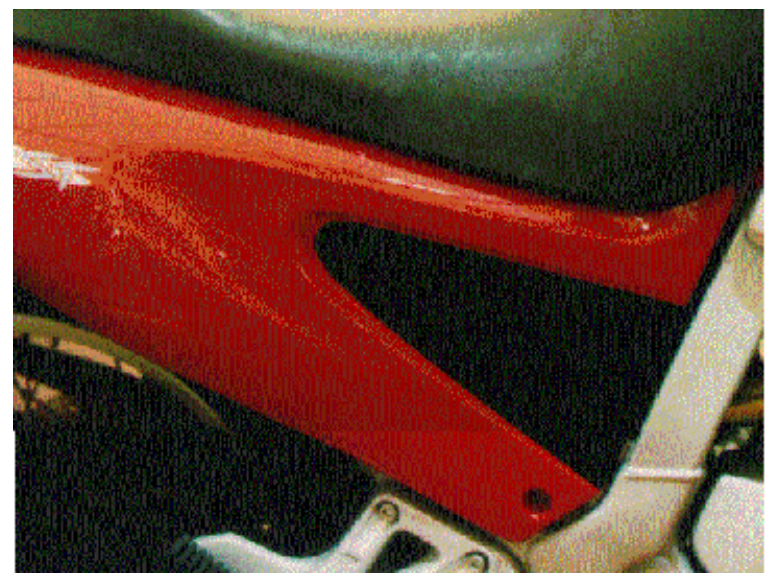

Fig. 13: Examples of compound features

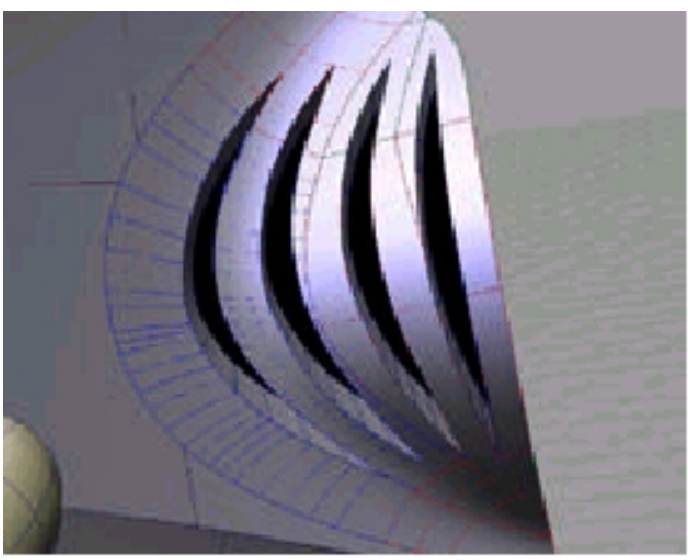

Fig. 14: An example of pattern-feature

\section{Types of simple features}

\subsection{Classification}

Going into the details of the shape representing the feature, we suggest now a classification describing elementary types of intrusions, extrusions and cuts according to the shape of the deformation produced and to the characteristics of the influence area and some evident lines which can be pointed out on the basis of the final aspect of the feature and can be used for shape modification purposes. We call these special lines as leading lines. Some of them can be associated directly to the character lines defined by the designer, while some other leading lines are curves emerging in a evident way because of a particular behaviour of the shape of the feature according to the intention of the designer. 
In the following tables we give a synthetic representation of the classification about elementary types of simple detail features obtained by intrusion, extrusion and cut which will be successively described.

In Fig. 15 we put into evidence elementary deformations according to the properties of the fundamental leading line (open or closed line) which is associated to each type.

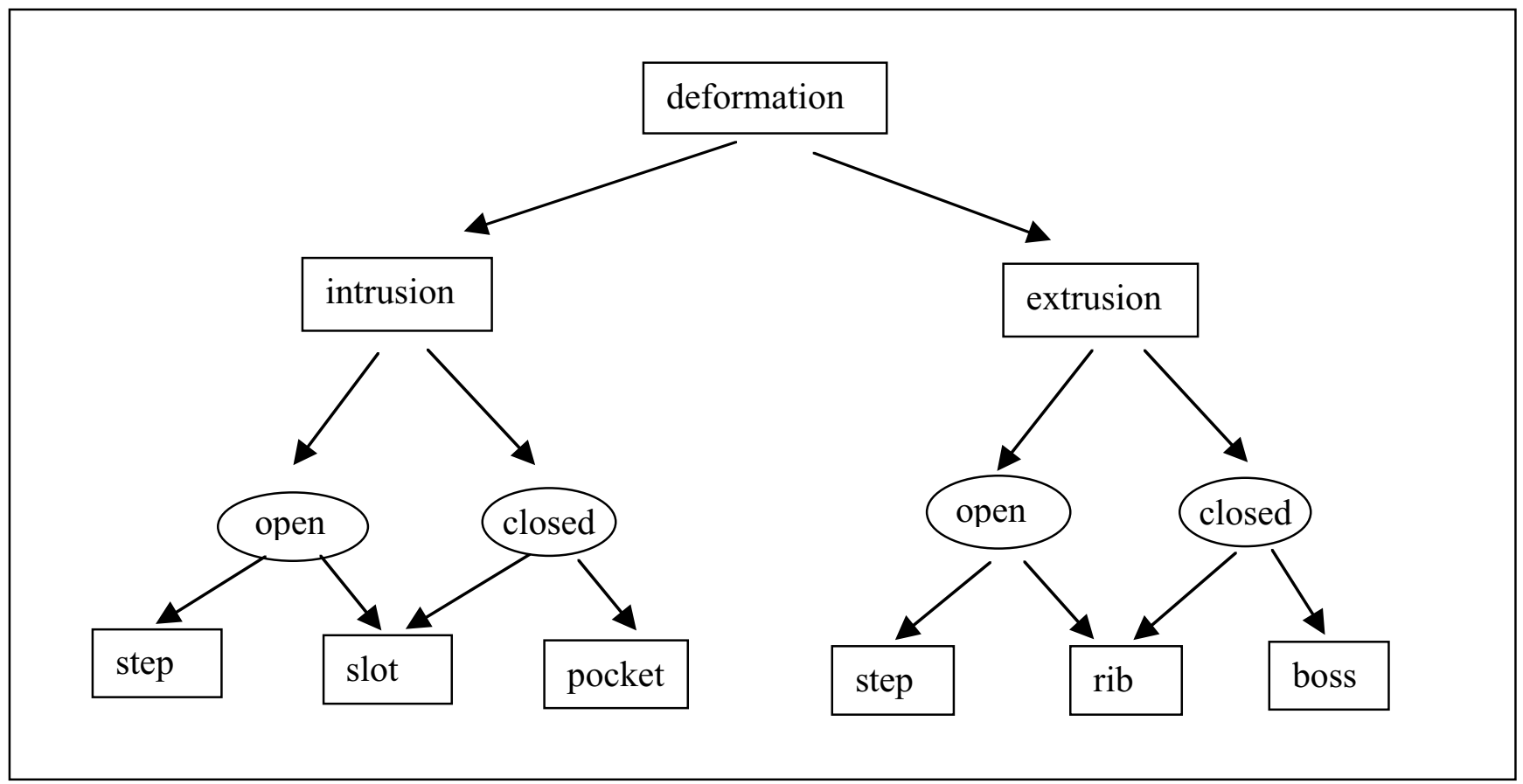

Fig. 15: Classification of elementary features obtained by deformation

In Fig. 16 the elementary types of cuts are listed according to the position of the influence area with respect to the surface boundary.

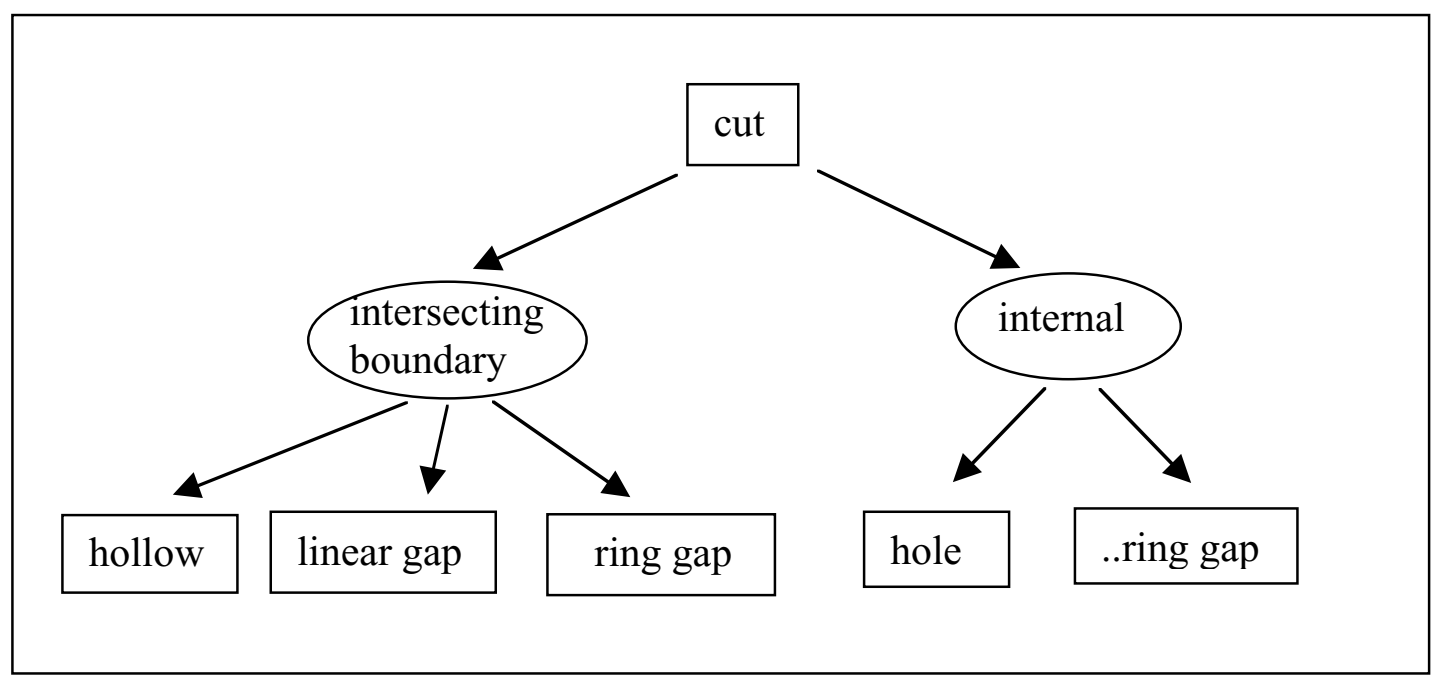

Fig. 16: Classification of elementary features obtained by cut 


\subsection{Types of simple deformations}

\subsubsection{Step feature}

It is an intrusion or extrusion which enforces the visual effect of an open leading line. Such line can be generally identified with the character line the stylist has designed to define aesthetically the detail feature. The influence area is prevailing from one side of that line and the deformation is strongly asymmetric with respect to the leading line defining the step.

A step feature gives the visual impression of a stair up or down ('sufficiently' smooth or sharp) over the surface, which corresponds to a protrusion or a depression respectively, what we will call a step up or a step down feature, depending on the modifications performed on the surface.

Displacement feature Step-like: If the effect of the step is obtained by raising or lowering a part of the influence area by means of a rigid translation, the feature corresponds to a special case of displacement: the so called step up or step down- by displacement. The characterized surface consists then of the displaced region and a transition surface. The curve bounding the displaced part from any transition surface is generally assumed as the leading line associated to the feature. The corresponding displacement area touches the influence boundary and produces a break over the part of the influence boundary intersecting the displacement area and over an adjacent part bounding the blending surface.

In Fig. 17 an example of a step feature is shown.

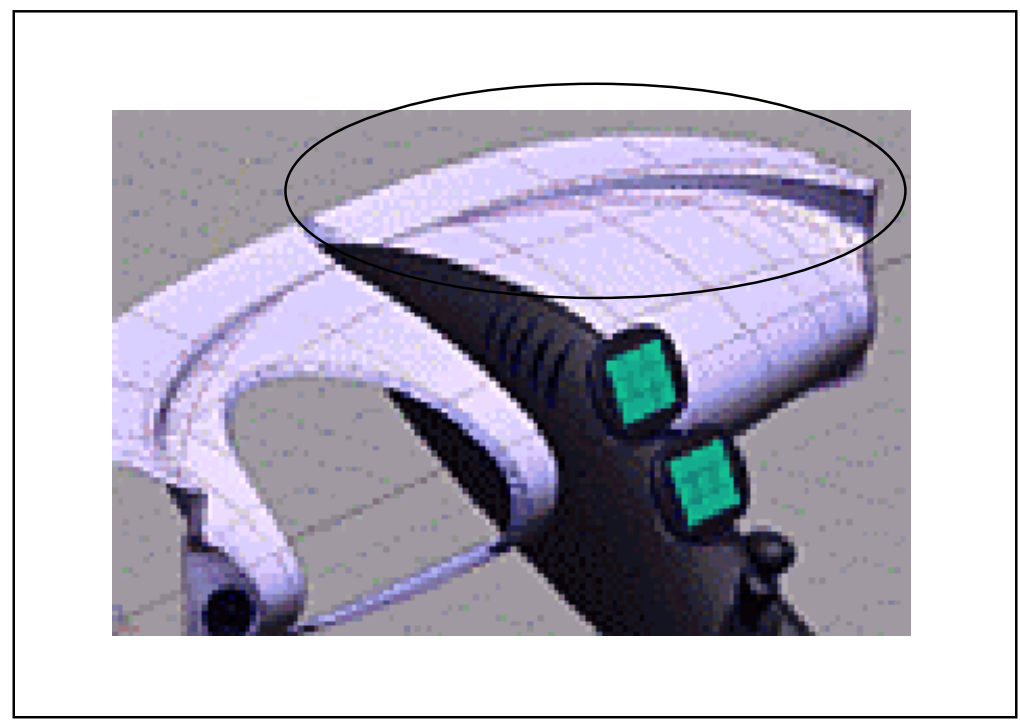

Fig. 17: Example of step feature

\subsubsection{Slot feature}

It is a depression having a shape with an evident longitudinal predominance and can be naturally associated to a line (the leading line of the slot). Thus the boundary of the influence area is located quite symmetrically at both sides of this line and in proximity to it. In most of the cases, the leading line is what is naturally designed by the stylist as the character line representing aesthetically a slottype detail. The leading line can be open or closed and the influence area can intersect or not the surface boundary. In case of a closed leading line, the influence area is a non simply connected region around it. The term is justified by that a slot feature has the appearance of a groove around the leading line. 
Because of the definition by means of a line and not of a region, features obtained by displacement of parts do not belong to this category. Details having the appearance of grooves with internal displaced parts have to be considered in the pocket type (see paragraph 4.2.3).

In Fig. 18 a detail representing a slot feature is shown.

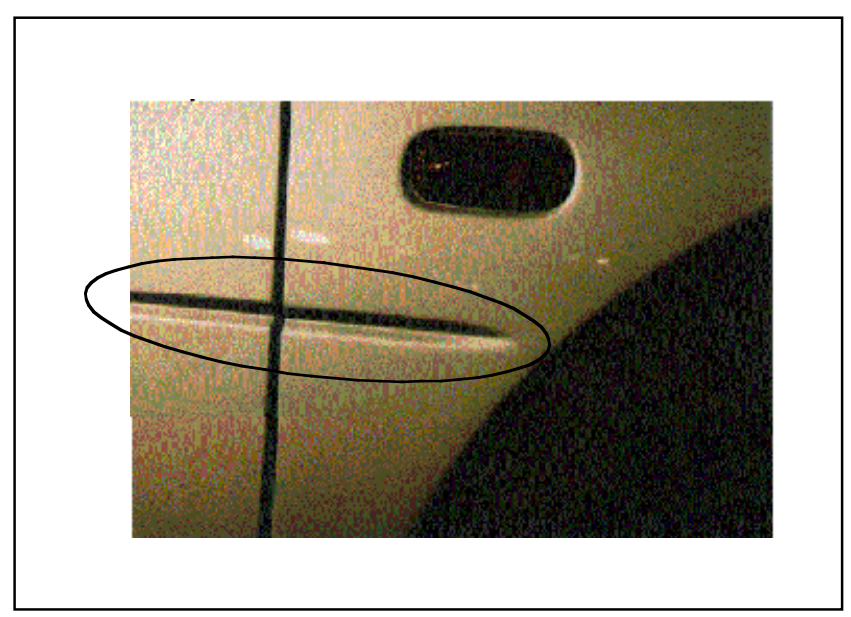

Fig. 18: Example of slot feature

\subsubsection{Pocket feature}

It is a depression inside a closed line. Differently from slot types naturally associated to a line, a pocket feature has an evident two-dimensional aspect, naturally associated to a region and, if a leading line is associated, it has to be closed in any case. Pocket types appear as cavities in which the intrusion has a sufficiently symmetric and uniform shape in both the degrees of freedom inside the influence area.

Displacement Pocket-like If a part of the deformed surface is obtained by a rigid translation of the area contained within the defining leading line, it corresponds to a particular type of displacement, which is called pocket feature by displacement. The displacement area has to be internal to the influence area (differently from step features by displacement in which the displacement boundary intersects the influence boundary producing breaks, i.e. $G^{-1}$ conditions).

An example of a pocket feature obtained over a surface is shown in Fig. 19.

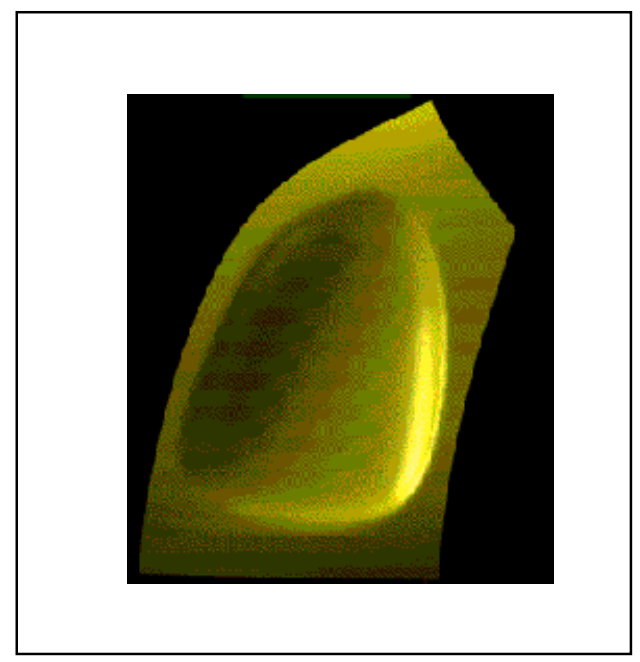

Fig. 19: Example of pocket feature 
In a similar way, we can define further elementary features which are obtained by extrusion.

\subsubsection{Rib feature}

It is a protrusion that appears like a nervation whose shape has a prevalent longitudinal aspect that can be seen as guided by a leading line such that the influence area is located around that line from both sides close to it. Properties of the rib features are the same of the corresponding intrusive slot type. Similarly to the latter, we do not consider a simple rib type obtained by displacement.

See Fig. 20 as an example of a rib feature.

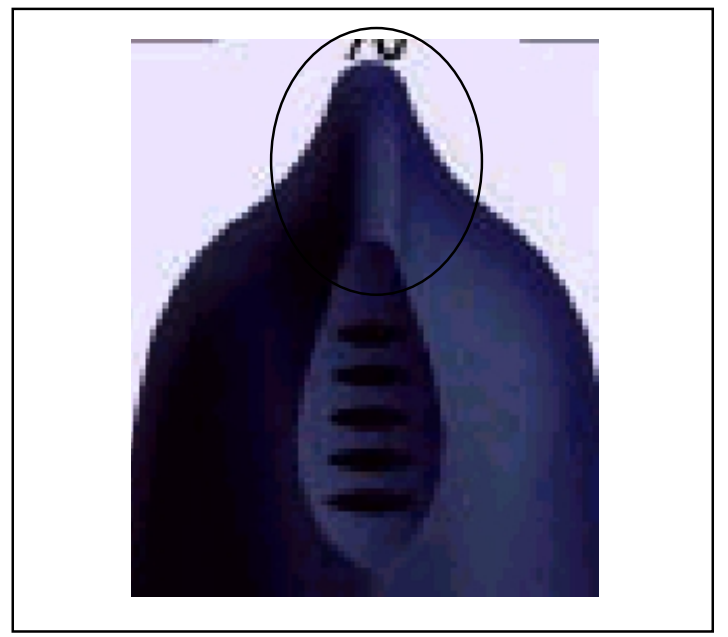

Fig. 20: Example of rib feature

\subsubsection{Boss feature}

It is a protrusion inside a closed line. It appears as a more or less extended convexity over the surface with a sufficiently symmetric and uniform shape inside the influence area and, if a leading line is defined, it has to be closed in any case.

Considerations analogous to the equivalent intrusive pocket type can be done. Particularly, while rib features are naturally associated to a line, the boss features are associated to a $2 \mathrm{D}$ region and have a sufficiently symmetric and uniform shape behaviour in both the directions. See Fig. 21 as an example.

Displacement Boss-like: In a way similar to pocket-by displacement features, in the extrusive case we can consider a boss by displacement (with or without break) where a part is obtained by rigid translation. Generally the curve separating the raised surface from the transition part, i.e. the boundary of the displaced surface, is assumed as the leading line associated to the feature. 


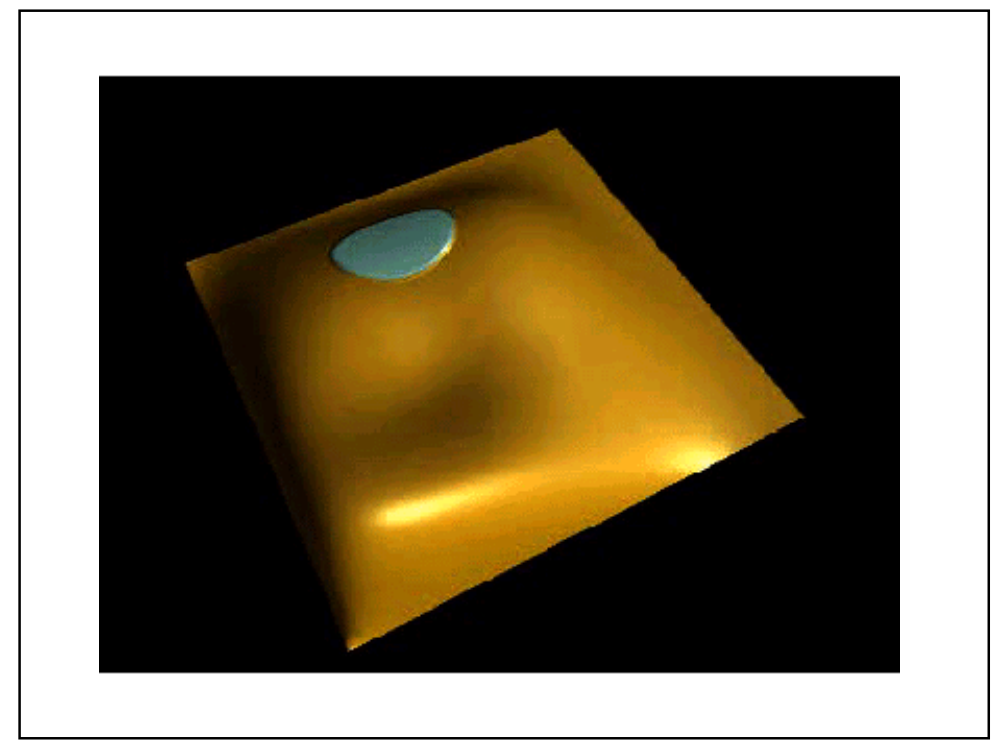

Fig. 21: Example of boss feature

It is worth observing that the distinction among the different types is not so evident. As an example, if the designer has to create a groove or a long narrow cavity, he can choose a slot or a pocket feature. In a similar way, there can be also doubts in the definition of a low large cavity not exactly symmetric or asymmetric as a pocket or a rounded step down type. In these particular cases the feature type is then singled out on the basis on the creation method, that is in the choice of the leading line and in the operative technique used for generating the feature and, finally, on the wished final aesthetic aspect. Thus, in the first example, the choice of a long narrow pocket instead of a slot depends whether the deformation has to be distributed in a quite uniform way inside the closed leading line, or it is mainly stressed around the corresponding open leading line. In the second case, whether a shape is defined as a non deep large pocket or a rounded step down type, it depends respectively again in the leading line respectively chosen as a closed or open curve, and also in the corresponding prevailing two-directional symmetry or one-directional asymmetry of the shape.

\subsection{Types of simple cuts}

Without lacking generality, it is possible to consider the surface to which the cut is applied as a simply connected region at least in an opportune neighbourhood (i.e. locally). We can therefore distinguish three cases:

a) the cut maintains both the local connection and the local simple connection (see Fig. 11a);

b) the cut breaks the simple connection but the local connection is left (see Fig. 11b);

c) the cut breaks the local connection and thus the simple connection (see Fig. 11c).

On the basis of this distinction, it is possible to define three elementary cuts, as described in the following sections. 


\subsubsection{Hollow cut}

It is a cut intersecting the boundary which does not produce disconnection (case a)). It appears like an indentation, an inlet at the surface boundary. The eliminated part is in any case a simply connected surface.

When the cut is sharp it is obtained by simple trim operations and its boundary corresponds to the influence boundary. If the designer defines the sharp hollow cut by a character line, it generally corresponds to its influence boundary, while in other cases, if the cut has to reveal a particular symmetry or regularity in the shape (i.e. hollows with longitudinal predominance), the character line could be an internal directrix curve around which the trimming is made.

In case of a finishing touch describing a smooth cut the trimmed area results contained within the influence area at least for the rounding. The semicircular indentation which is applied to the arch wheel derives from an hollow cut, as shown in Fig. 22.

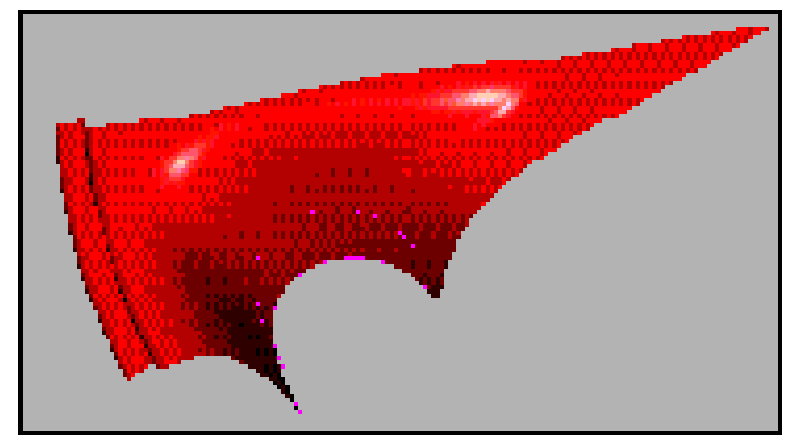

Fig. 22: Example of hollow cut.

\subsubsection{Hole cut}

It is an internal cut which yields a surface which is not simply connected whichever neighbourhood is considered (case b)). The eliminated part is a simply connected region topologically equivalent to a hole.

In case of a sharp cut also holes can be obtained by simple trimming the primary surface in the influence area, and the closed influence boundary, which does not intersect the surface boundary, can correspond to the designed character line. Otherwise, a regular behaviour of the shape or particular symmetries could be expressed by designing the character line originating the sharp hole as an internal directrix curve.

In case of a finishing touch describing smooth holes, further surfaces can emerge because of bends adjacent to the cut due to fillet operations.

An example of hole cut is shown in Fig. 23. In this case the cut involves two different patches. 


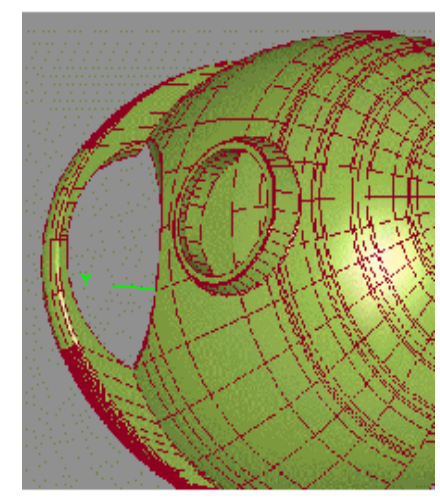

Fig. 23: Example of hole cut

\subsubsection{Gap cut}

It is a cut which produces a disconnected surface (case c)), i.e. it gives raise to two parts totally separated. Depending on the property of simple connection for the eliminated part, we can distinguish two types of gaps: the linear gaps, in which a simply connected part is eliminated (necessarily intersecting the surface boundary), and the ring gaps, in which the eliminated part is a ring with circular topology (which can intersect or not the surface boundary).

Generally a gap has intrinsically a longitudinal predominance and it is usually inserted in correspondence of the conjunction of different product parts, like the car doors (linear gap) or the petrol cap (ring gap), thus it can be meaningfully associated to a character line having both functional and aesthetic impact. The character line is generally designed as the directrix curve expressing the longitudinal behaviour of the gap. Otherwise, it can be directly associated to the gap boundary if there is not an evident regularity in the shape. The boundary of a sharp gap, i.e. the trimming curve, can be:

- a connected loop of curves, partially overlapped with the surface boundary (linear gap);

- constituted by two closed connected loops of curves, one internal to the other, non crossing the surface boundary (internal ring gap);

- constituted by two connected loops of curves, one open and the other closed, the former crossing the boundary (non internal ring gap), having one piece inside the other one.

Also in case of smooth gaps, finishing touches consisting in bending and fillet operations can let emerge surface lines adjacent to the trimming curves.

In Fig. 24 a ring gap and a linear gap are shown. 


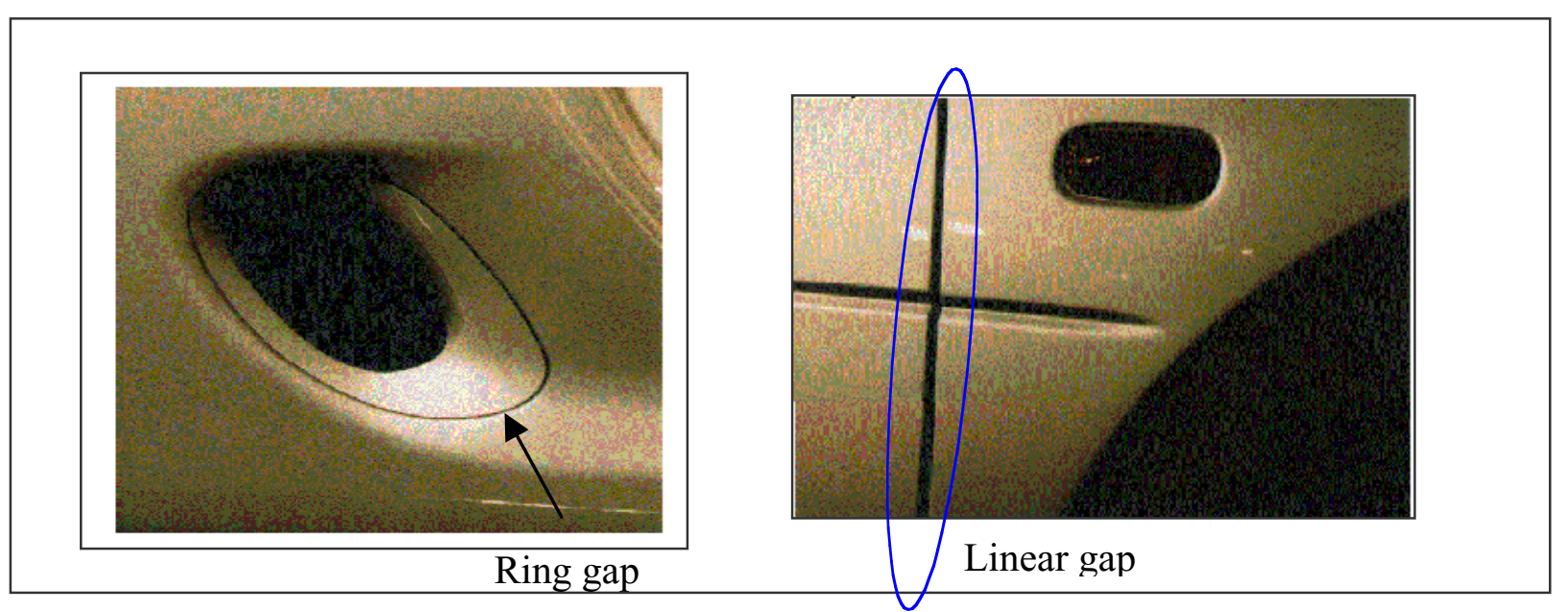

Fig. 24: Examples of a ring gap and a linear gap

\section{Definition of geometric parameters}

In this chapter we describe some geometric parameters used for the definition of feature classes. The purpose is to define the information that has to be known to characterize the shape of the detail features. The list of such information and parameters has to be utilizable for the implementation, in order to create features by modelling surfaces at the computer.

It has to be noticed that the parameters have been chosen mainly thinking of features obtained by means of deformation functions [18, 19], therefore different/additional parameters could be given depending on the implementation aspects. Using a predefined list of already existing modelling operators it might be necessary to consider further parameters required by the operators used.

Let us point out the basic parameter types.

\subsection{Essential parameters}

\subsubsection{Leading lines}

In aesthetic design it is important to have the possibility of creating arbitrary shapes and modifying them according to the creativity of the stylist. In order to control the shape of the form features over a surface, a possibility is to use special curves which could define the shapes and emphasize them in an aesthetic sense.

A leading line corresponds to the curve chosen by the stylist for driving the shape of the deformation. This is done by giving an initial curve $\gamma$ (leading line on the primary surface) and specifying the target curve $\gamma^{\prime}$ which represents the shape of the leading line when projected onto the resulting deformed surface according to the given deformation law (see chapter 3 ).

Some of the leading lines may correspond to the character lines of the product. This happens when they give an evident visual and aesthetic impact to the surface and are assumed as reference lines used to define aesthetically the detail feature. In fact a character line is distinguished by other lines which can be singled out over a surface because of these two main aspects:

- aesthetic evidence: the character line gives an immediate aesthetic characterization to the product; 
- operative role: the feature is designed starting from a character line which represents its first definition parameter of the feature.

A leading line is defined as a curve (possibly a set of adjacent curves) $\gamma$ over a surface. From an operative point of view a leading line can be defined as it follows. Let us consider a primary surface $S$ defined with respect to a fixed reference system $O x y z$, and a curve $\gamma_{0}$ (called basic leading line) whose position is known with respect to the surface. The leading line $\gamma$ is then obtained by projecting the curve $\gamma_{0}$ onto the surface, i. e. the curve $\gamma=p\left(\gamma_{0}\right) \subseteq S$ ( $p$ being the identity function if the curve $\gamma_{0}$ already belongs to the surface).

The determination of the leading curve from the basic one will be referred in the following taxonomy description (see chapter 6) as the projection law for the basic line.

The possibility of starting from a curve not belonging to the surface is necessary for comprehending the case in which leading lines are defined as curves originated by lines lying elsewhere. In fact it commonly happens that the designer is interested in reproducing the same shapes from one part of the object to the other, or for different objects (think of lines and symbols defining products belonging to the same trade-mark, like the typical 'logo' defining the producer company).

We can then define the target leading line as the curve corresponding to the leading line in the deformed surface, that is the curve $\gamma^{\prime}=\left\{P^{\prime}: P=\delta(P), P\lfloor\gamma\}\right.$, where $\delta: S \diamond R^{3}$ is the deformation law (see Chapter 6). In Fig. 25 the relations among the basic leading line, the primary leading line and the leading line are shown.

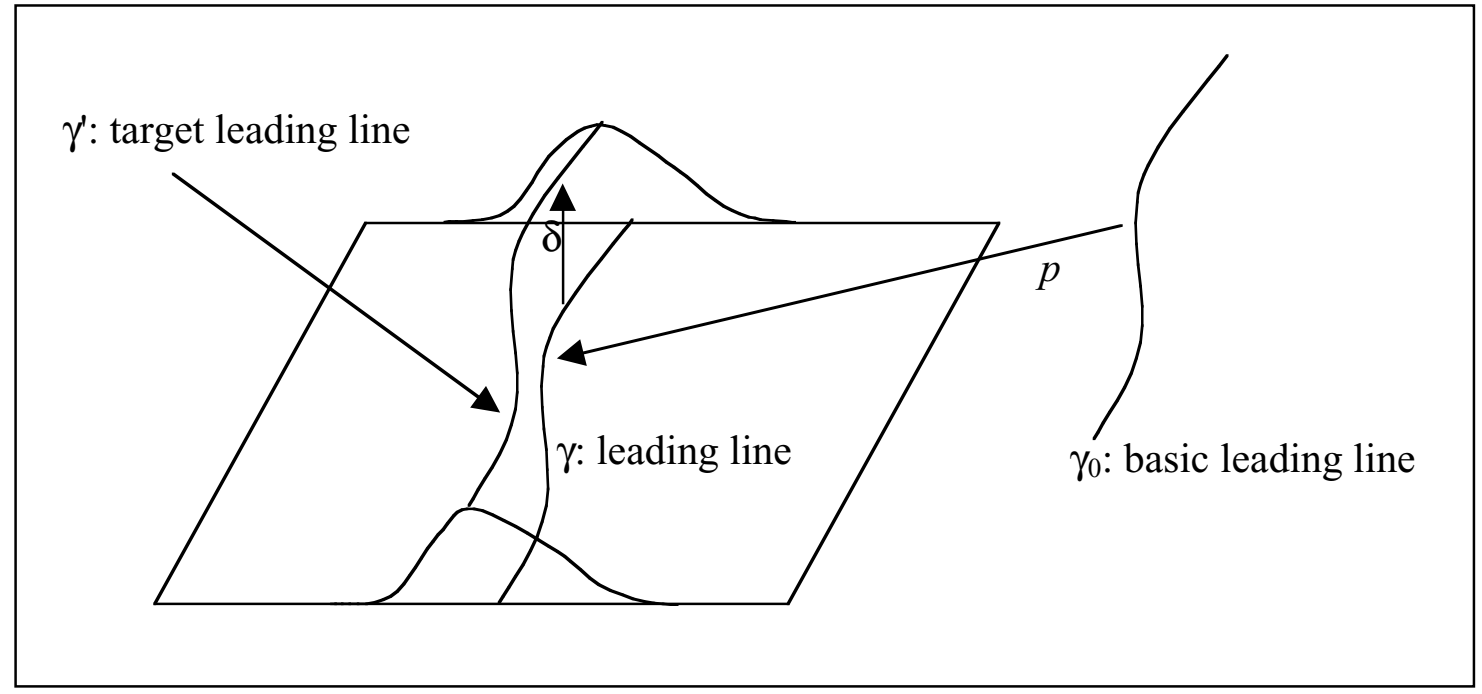

Fig. 25: Representation of the leading lines $\gamma_{0}, \gamma$ and $\gamma^{\prime}$

A leading line can be an open or closed line. For aesthetic reasons, it is required that the curve has a certain level of regularity to be specified.

We lay stress on the fact that leading lines are a different concept from the influence boundary. Even if sometimes they can coincide, as in the case of a sharp cut consisting in an elimination of the region inside a closed line, usually a leading line is strictly contained within the influence boundary. 


\subsubsection{Influence area}

As already said, the influence area is the region of the primary surface to be modified by the insertion of a feature. The extent of it is specified by the definition of its boundary.

Such definition can be done in different ways:

- Directly by the assignment of curves representing the boundary of the influence area. Operatively this can be done by projecting onto the primary face the boundary drawn on an help plane similarly to the process explained in the previous paragraph for the definition of the leading line from the basic one.

- By deriving curves from the leading lines according to specific criteria, like considering positive real numbers, or positive functions which establish the size of the influence area around the character line.

In figure 26, examples of influence areas derived from the leading line at non constant distance are given. In the examples a slot feature characterized by a leading line $\gamma_{1}$ is considered, where the influence area is derived by using two functions $\alpha_{1}(s)$ and $\alpha_{2}(s)$, being $s$ is the arc length of the curve $\gamma$ : they represent the distances $\overline{P P_{\gamma}}$ (constant or non constant) of points $P_{\gamma}$ lying on the leading line from P projection-point of $P_{\gamma}$ on the influence boundary on one (or on the other) side of the leading line along a fixed projection direction. If $\alpha_{1}(s)=\alpha_{2}(s)$, the slot produce a symmetric deformation along the curve $\gamma$. In addition, since the deformation area has to correspond to a closed region of the primary surface, additional criteria for closing the area should be considered. In the examples shown a possibility could be to consider the curve having minimal length and passing through the extremes of the three curves.
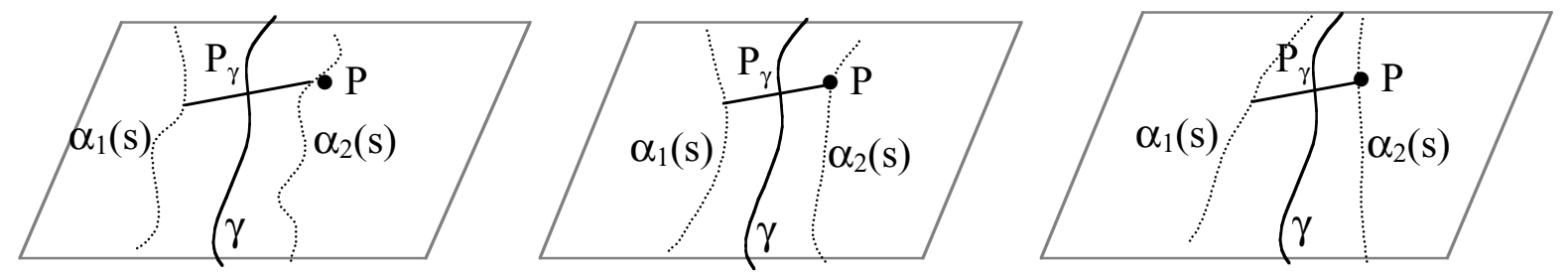

Fig. 26: Examples of functions defining the width of the influence area around a leading line

For sake of simplicity, in the following taxonomy specification we will indicate with the general term influence boundary generation law the way used for creating the curves defining the boundary. This law includes also all the values necessary for the boundary generation, e.g. for the direct assignment of the curves the equation of the help plane and the projection direction.

\subsubsection{Depth}

The deformation law of the leading line into the target one in special cases may be expressed by quantitative parameters. In particular, the level of incidence and impression over the surface caused by the feature can be quantitatively expressed by a parameter describing its depth. We can define the depth as the vector projection of the generic point belonging to the leading line over the initial surface onto the corresponding point over the characterized surface. 
The depth expresses the generation law of the target leading line over the modified surface. As a vector, depth can be given by:

- an oriented direction establishing if the target leading line locally lies on a depression or a protuberance (if the direction is concordant with the surface normal then we have a protuberance, else a depression);

- an intensive scalar value, displaying how deeply the leading line engraves on the surface.

We can assign depth which varies in intensity and direction (or both) along the leading line. In this case, given on oriented direction along the curve, depth is expressed as a vectorial function $\mathbf{h}(s)$ defined for each point of the primary leading line $\gamma_{1}$ having arc length $s$. Consider that the variation of depth has to maintain a certain degree of continuity in order to avoid unexpected or not enough regular shapes.

In Fig. 27 the vectorial function $\mathbf{h}(s)$ defined over the leading line is displayed.

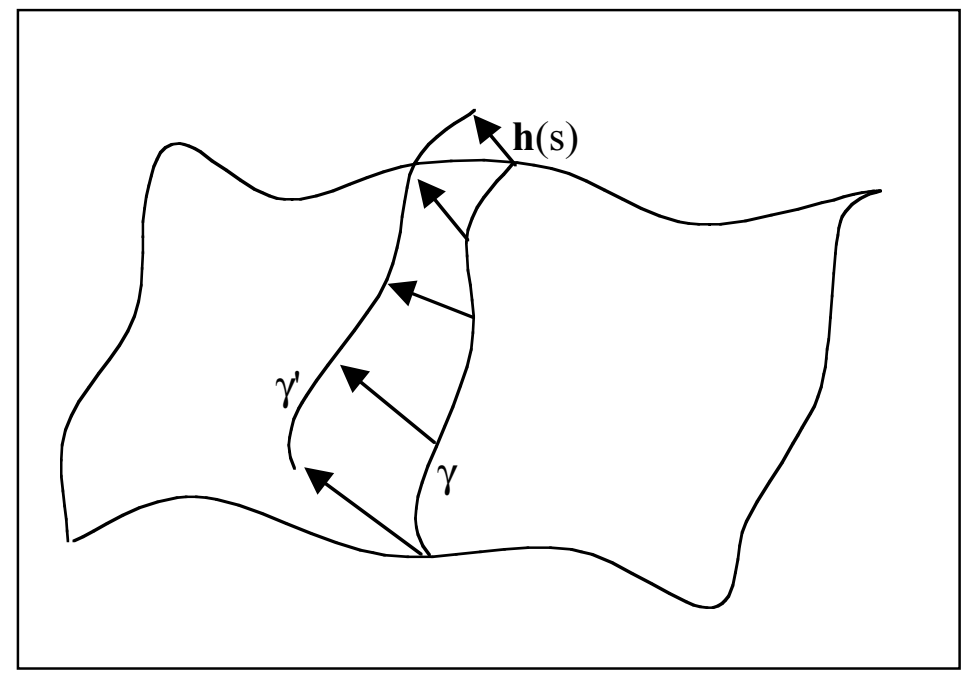

Fig. 27: Depth function associated to the leading line

\subsubsection{Transition conditions}

The designer must have the possibility to decide the type of transition between the modified surface and the unmodified part. Transition conditions are expressed by the so-called geometric continuity $G^{n}$ conditions [2] along the influence boundary. It has been defined that:

Two surfaces join with geometric continuity $G^{n}$ along a fixed curve if there is analytical continuity $C^{n}$ in at least a surface neighbourhood with respect to an opportune parametrization of the resulting joined surface.

Let us see the practical meaning of the most used geometric continuity conditions.

Practically only $G^{-1}, G^{0}, G^{1}$ or $G^{2}$ continuity are treated by CAD/CAS systems. A $G^{-1}$ continuity describes formally the idea of discontinuity, that is a jump between the modified and the unmodified regions in proximity of the considered curve, like in case of deformations with break. $G^{0}$ continuity expresses the simple continuity among adjacent parts. With regard to this we point out that for aesthetic purposes usually the $G^{0}$ continuity is not enough since it introduces a visible edge unless it has to enforce the effect of a union of two surfaces. $G^{l}$ continuity describes a soft bending having continuity for the tangent. Finally, $G^{2}$ continuity requires also continuity in curvature. 
We report in Fig. 28 the emphasized visual representation of these types of geometric continuity among two non parallel planes.

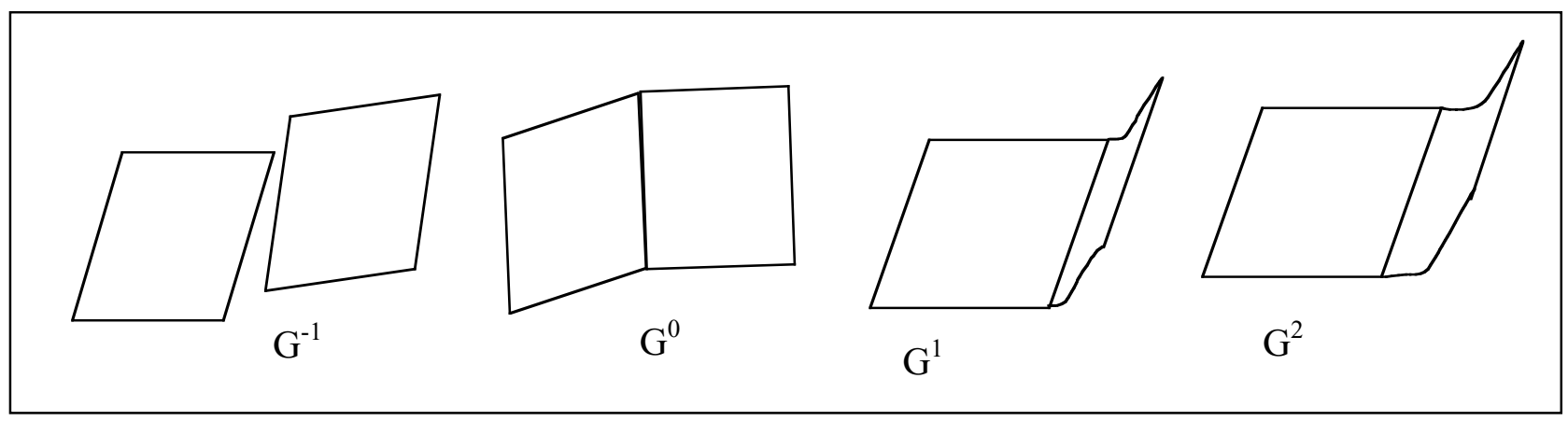

Fig. 28: Examples of the most common types of geometric continuity.

\subsection{Shape control parameters}

The parameters we have just mentioned give the basic structure to describe a lot of local surface deformations representing detail features.

At this point the designer could have the necessity to specify also some other parameters in order to characterize the shape of the modified surface for a more direct shape control of the feature. Some of them are here suggested.

\subsubsection{Leading points}

This information is meaningful especially for features not strongly characterized by a curve, e.g. pockets and bosses, for which it could be useful to drive the modification in the shape by only means of set of leading points distributed in the influence area. In such a case, instead of fixing a continuous vectorial depth function describing the generation law of possible target leading lines, it is possible to give a discrete set of applied vectors $\left(P_{i}, \mathbf{h}_{i}\right)$, for $i=1,2, \ldots, n$, where each point $P_{i}$ belongs to the primary surface inside the influence area and $\mathbf{h}_{i}$ are the corresponding projection vectors applied by each of the points $P_{i}$, such that $P_{i}^{\prime}=P_{i}+\mathbf{h}_{i}$ defines the target leading points $P_{i}^{\prime}$, for $i=1,2, . ., n$, through which the modified surface has to pass (by interpolation or approximation). The current version of the FdF operator from Matra DataVision [17, 18], is based on this idea.

We have to verify that the leading points $P_{i}$ lye inside the influence area and that there is consistency with the possible known information about the depth of the target leading line, if previously defined, in case any point $P_{i}$ belongs to the leading line.

In Fig. 29 the discrete system of depth vectors $\left(P_{i}, \mathbf{h}_{i}\right)$ characterizing the shape of an extrusion is displayed. 


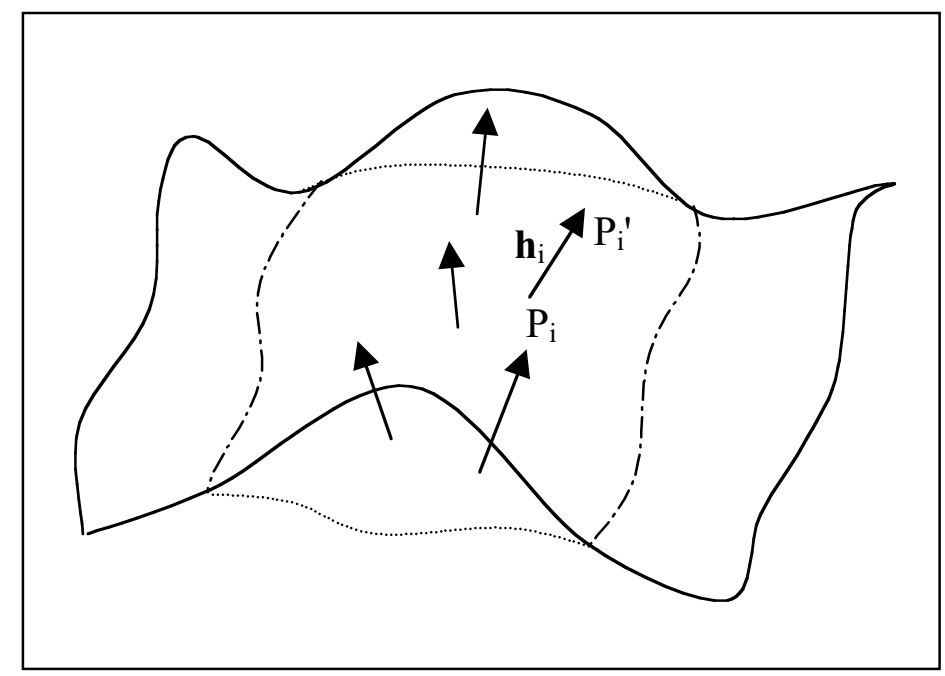

Fig. 29: Discrete set of target leading points and corresponding depths

\subsubsection{Sections}

The resulting shape of the deformed surface could be also constrained by imposing the passage through given curves defining plane sections of the feature. For example, this could be done by assigning in input a family of $n$ planar curves, for $i=1,2, . ., n$, having a parametric representation $\left(X_{i}(t), Y_{i}(t)\right)$, for $a_{i} \leq t \leq b_{i}$, over planes $\pi_{i}=(X Y)_{i}$ with known position with respect to the surface. Planes could be located providing values for a point $P_{i i}$ of the plane and the normal unit vector $\mathbf{n}_{i}$.

Practically section-curves are assigned along special directions. For this purpose, some concepts of differential geometry are specified:

Given a surface and a curve contained in it, for each point $P$ belonging to the curve we define transversal plane in $P$ the plane passing through $P$ which is perpendicular to the tangent unit vector at the curve in $P$.

Such plane (existing and univocally determined for each point, since we consider regular curves) contains the normal and binormal unit vectors of the Frenet trihedron of the curve in the considered point, and it is perpendicular at its osculating plane. Besides:

Given a surface and a curve contained in it, for each point $P$ belonging to the curve we define transversal profile in $P$ the (planar) curve passing through $P$ which is obtained as section of the surface with the transversal plane in $P$.

Later on, some special cases of sections are then described.

Transversal profiles: A possible way for describing the shape behaviour of the feature around the leading line could be provided by fixing transversal profiles passing through $n$ points $P_{i}$ belonging to the leading line. In this case profiles belong to planes which are not in general parallel.

It is evident that constraints of compatibility with the previous parameters have to be respected. Profiles have to intersect the leading line and have to satisfy the given geometric continuity conditions at the corresponding points belonging to the boundary influence. 
If a feature is described by several leading lines, then it should be possible to assign different sets of profiles in proximity of the different lines, if existing. Of course, if planes containing such profiles are coincident, common pieces of profiles have to be equal and superimposing.

Vertical parallel profiles: Another choice for describing the shape behaviour of the feature around the leading line $\gamma$ can be to assign section-curves on planes having a fixed direction which is concordant or coincident with a 'representative' normal unit vector at the surface, and this direction can be considered in any case as vertical with respect to an opportune reference system. In this case vertical sections belong to parallel planes (so that they can be called vertical parallel profiles) and analogously to the case of transversal profiles, we need to verify compatibility of the shape of each curve with the given geometric continuity conditions.

Like in case of transversal profiles, it is possible to assign different sets of profiles in proximity of different leading lines if existing.

Contour lines: Especially in case of intrusions and extrusions having complex shape, it could also be possible to constrain the feature shape by considering horizontal sections (with respect to an opportune reference coordinate system) for given altitudes $h_{i}$, as it is often used in topography. This corresponds to the definition of a family of contour lines describing the set of points at the same height, according to an opportune reference coordinate system. In this case we have to assign closed or open lines disjoint among them, and disjoint with the projection of the influence boundary onto the same horizontal plane.

However, if a leading line exists, instead of working by contour lines on the horizontal plane, the designer tends to prefer a definition of vertical parallel profiles or transversal profiles, in order to have a more immediate visual representation of the feature shape to be built around the leading line.

In Fig. 30 we represent the three types of plane sections we have just described.

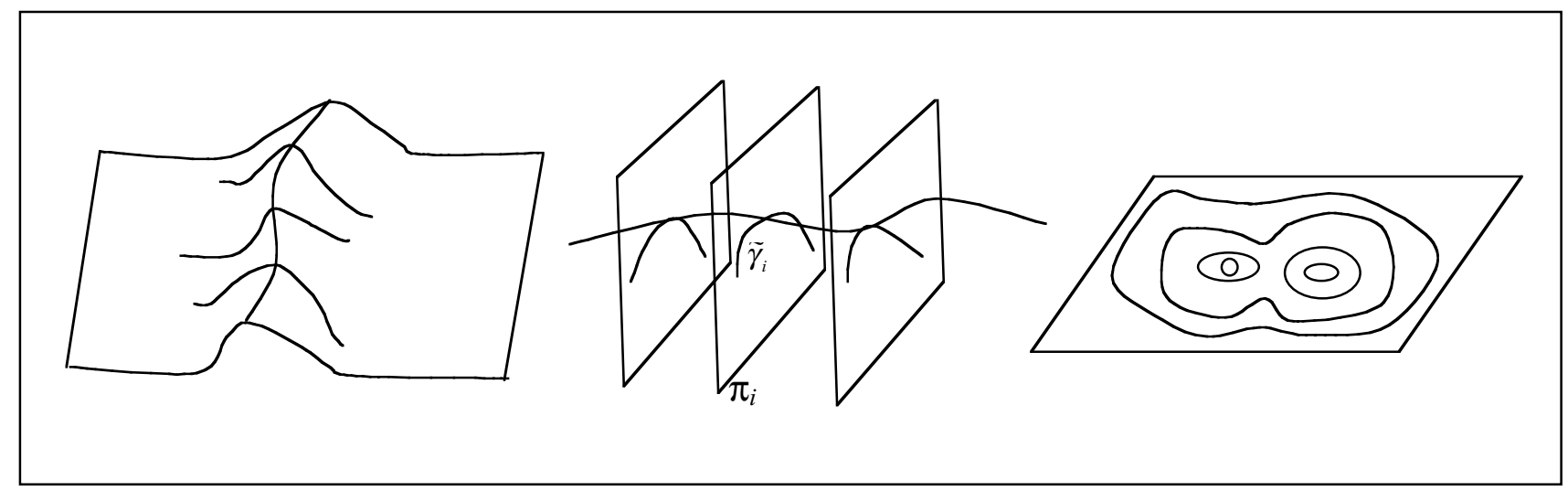

Fig. 30: Transversal profiles, vertical parallel profiles and contour lines

Once these curves are assigned together with the corresponding leading lines, the feature should be obtained as a surface (for example NURBS surfaces) approximating or interpolating such lines consistently with the other definition parameters of the feature.

This group of information has the advantage that the final shape of the feature is well-controlled, because information is global (since the knowledge of a curve is global), but the drawback is that it is necessary to verify the previously considered compatibility conditions. 


\subsubsection{Curvature and gradient laws}

Another possibility for controlling the shape of the feature can be provided by considering the degree of bending for the surface-feature along opportune directions. These directions can be chosen in relation to the leading line because of its privileged role. Regarding to this, let us define some concepts from the notion of curvature [2] and transversal profile.

Given a curve belonging to a surface and a point $P$ of the curve, we define transversal curvature of the curve in $P$, the curvature $k$ of the corresponding transversal profile in $P$. We define then vectorial transversal curvature the vector $\mathbf{k}=k \mathbf{n}$, whose intensity is the transversal curvature, and whose direction is the normal unit vector $\mathbf{n}$ of the transversal profile in $P$.

Knowing the vectorial transversal curvature is equivalent to knowing the scalar curvature together with the tangent vector $t$ or, analogously, the radius $\rho$ and the centre $C$ of curvature, $i$. e. knowing the osculating circle of the transversal profile in $\mathrm{P}$.

Therefore, instead of giving global information like profiles (see paragraph 5.2.2), it could be meaningful to assign information about curvature along special directions over the surface to be modified. Precisely, if we consider the arc length s of the leading line, it is possible to define a scalar transversal curvature function $k(s)$, or assign the vectorial transversal curvature function $\mathbf{k}(s)$ (transversal curvature law) or, equivalently, the curvature radius function $\rho(s)$ and the tangent vectorial function $\mathbf{t}(s)$, or he could give only $\mathbf{t}(s)$ (the last will be called transversal gradient law).

In an analogous way, instead of transversal curvature and gradient laws, we can define curvature and/or gradient laws for vertical parallel profiles along a fixed direction. Besides different curvature and/or gradient laws can be given for different leading lines, if existing.

In Fig. 31 we give a representation of curvature and gradient laws we have just described.

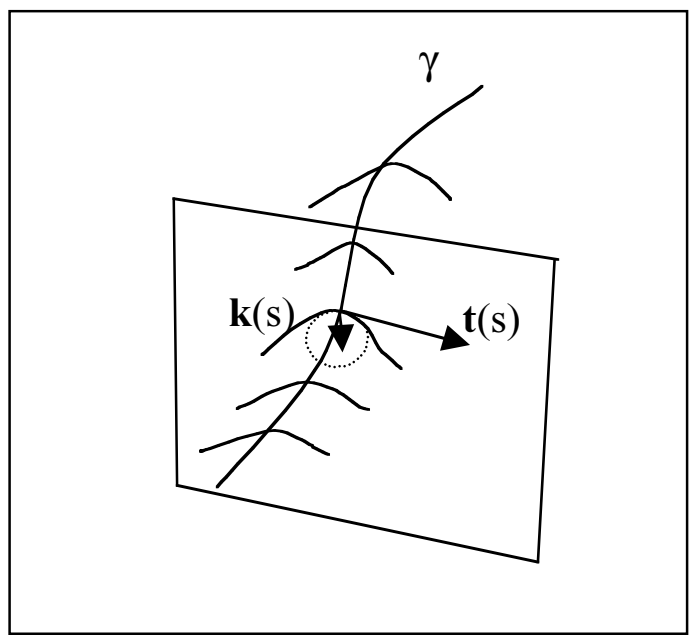

Fig. 31: Transversal curvature and gradient laws

In both cases it is evident that this group of parameters give a local type of information about the feature surface (since concepts of curvature and tangent vectors, etc. are local), leaving still degrees of freedom in the shape of the feature (coherently with the other given parameters). It is necessary to verify the consistency with the shape of the profiles, if they are assigned on the planes crossing the leading line at the same values of its arch length as further parameters along the same direction of vertical planes for which curvature and/or gradient laws are given. 
A drawback seems to be the operative difficulty of expressing curvature and gradient laws in a form which is useful for the implementation at the computer. For this reason, generally discrete values of curvature and gradient at selected points of the leading line are preferred to information given by functions defined over continuous sets. For example, transversal or vertical parallel curvature or gradient laws could be given by means of vectors $\mathbf{k}_{i}=\mathbf{k}\left(s_{i}\right)$, or values $k_{i}=k\left(s_{i}\right)$ i. e. equivalently the curvature radii $\rho_{i}=\rho\left(s_{i}\right)$, and/or tangent vectors $\mathbf{t}_{i}=\mathbf{t}\left(s_{i}\right)$, where $s_{i}$ are the values of the arc length of the leading line corresponding to a selected choice of points $Q_{i}$, for $i=1,2, \ldots, m$, over the line.

The parameters above suggested represent only one among several possible choices, and they correspond to the theoretical meaningful parameters defining the deformation function. At this level, there is no reference at the conversion in a operative form which can be used for implementation.

Once these parameters are defined, it is possible to present a formal classification of features on the basis of the type, the hierarchical relations, the essential and shape control parameters, the constraints and the compatibly conditions to be respected.

\section{Feature classes}

\subsection{Definition of a class}

In the proposed feature classification (see Fig. 32) we identify the generic detail feature as the general abstract class in which parameters are information about the domain, the deformation law itself (virtually any law) and the attachment relations between the modified and the unmodified parts. The first lower classes, derived from the general detail feature class, are the abstract types intrusion, extrusion and cut, from which the derived classes are defined by considering the feature specific parameters and constraints among them.

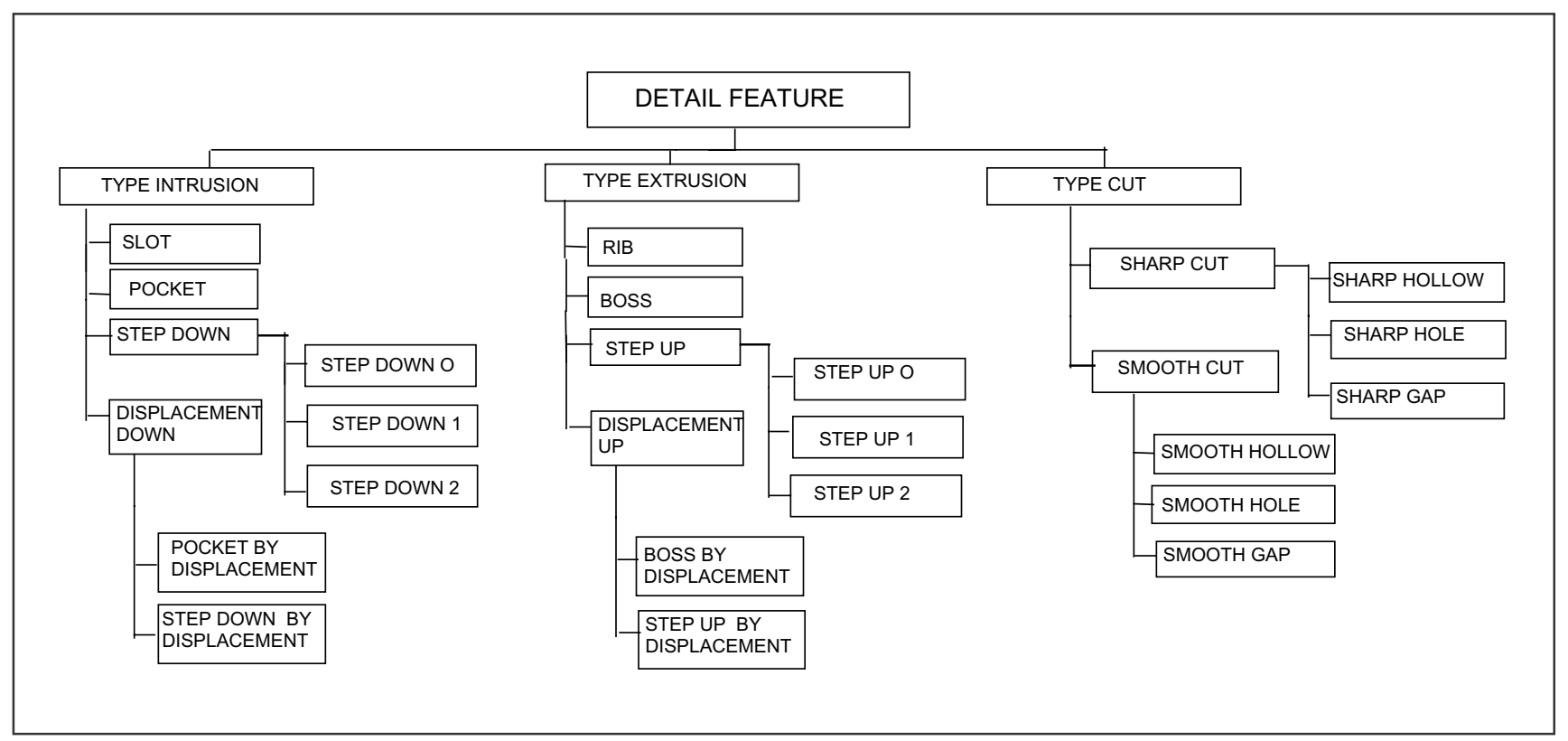

Fig. 32: Feature taxonomy 
In the next paragraph each feature-class is defined and described, according to the following scheme:

Derived from: general class from which the feature is derived as a particular class

Name: $\quad$ term identifying the specific feature

Definition: short description of the feature

Parameters: geometrical and topological entities necessary to define a particular shape (if the class is derived such parameters have to be considered together with those ones already listed in the upper class they belong to)

Constraints: conditions and restraints that parameters have to satisfy

It is worth observing that the suggested feature classification is not exhaustive, since the described non abstract classes represent only a set of possible simple detail features. More specific subclasses could be derived from the considered classes, in which distinction could be given on the basis of specific geometric properties having the shape of the feature, like particular symmetries in the characterized surface and regularities of the leading line (if existing). For example, basic leading lines could be given as straight open lines or circles, the depth of the target leading line could be constant, the influence area could be the projection of a regular $2 \mathrm{D}$ geometric figure onto the primary surface, profiles could have regular shape, and further similar characterizations.

In the classification that follows a special stress is laid on some particular displacement recalling the appearance of step, pocket and boss features.

In addition, various specific classes derived from the step up and step down features (see Fig. 33) are also considered, according to the properties of the generation law of the target line from the leading line originating the step. The distinction among specific types of step up and step down classes is done by considering three possible cases: both the extremes of the leading line for generating the target line are moved (step up/down 0 ), or only one (step up/down 1 ), or both the extremes remain fixed (step up/down 2).

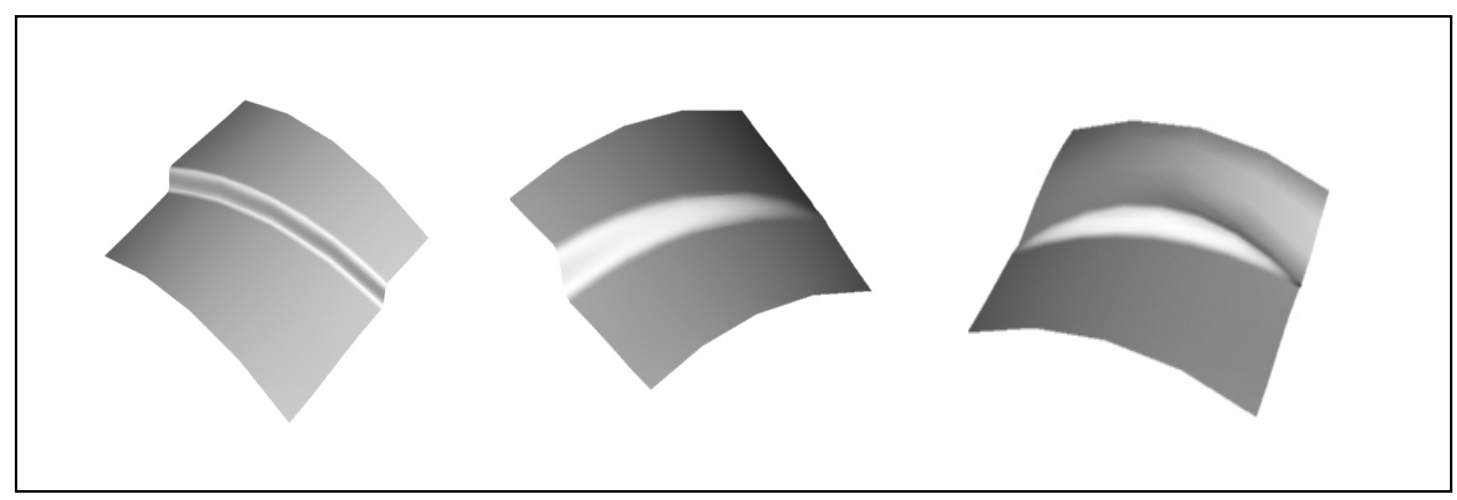

Fig. 33: Examples of step up features of type 0,1,2

In the following description any reference to concept of concavity, maximum/minimum and inflexion points is intended with respect to an opportune 2D reference coordinate system xy, containing the considered plane curve, in which the y-axis is chosen in the same direction of projection of the leading line onto the target line. 


\subsection{Feature classes}

\section{DETAIL FEATURE}

Derived from: Abstract - (general upper class)

Name: Detail feature

Definition: Result of a local modification of a free-form surface

Parameters:

- Primary surface $S$

- Curve representing the influence boundary

- Influence boundary generation law (1)

- Transition conditions along the influence boundary between the modified and the unmodified part of the surface (geometric continuity conditions $G^{-1}, G^{0}, G^{1}$ or $G^{2}$ )

- Surface deformation law (2)

Constraints on Parameters:

- Non enveloped primary surface

- Connected primary surface

- Influence area ( influence boundary) contained in the primary surface

- Connected influence area

(1) This can be necessary since the influence boundary can be created in several ways, e.g. by considering the projection of a closed loop of curves from a convenient plane onto the primary surface, or derived from some computations (see chapter 5).

(2) Possibly hardcoded as a sequence of basic modeling operations, or using energy criteria, etc. i.e. the modeling operations generating the surfaces of the feature depending on the type.

(3) Any reference to concept of concavity, maximum/minimum and inflexion points is intended with respect to an opportune $2 D$ reference coordinate system xy, containing the considered plane curve, in which the $y$-axis is chosen in the same direction of projection of the leading line onto the target line

\section{TYPE INTRUSION}

Derived from: Abstract - Detail Feature

Name: $\quad$ Intrusion or depression type

Definition: Detail shape obtained by a surface deformation having discordant direction with respect to the normal unit vector at the surface for each point belonging to the influence area

Parameters:

- Curves representing the leading basic line

- Relative position of the leading basic line and the influence area as regards the surface

- Projection law for the leading basic line

- Deformation law for the generation of the target leading line

- Shape control conditions

Constraints:

- Non enveloping leading line

- Leading line contained in the influence area

- Law for generating target leading lines such that depth has direction discordant with the normal unit vector at the surface

\section{Examples of possible shape control conditions:}

\section{Leading points:}

Parameters:

- Point co-ordinates in the primary surface

- Target position for the points (vector depth)

Constraints:

- Points belonging to the influence area

- Vector depth of target points discordant with the normal unit vector at the surface 
- For any point belonging to the influence boundary: it must exist a $G^{-1}$ condition set in a neighbourhood of the point

\section{SLOT}

Derived from: Intrusion type

Name: $\quad$ Slot feature

Definition: $\quad$ Surface representing an intrusion in a neighbourhood of a target leading line, such that in the domain over the influence area each transversal profile at the line in each point locally has a concavity towards a direction concordant with the surface normal unit vector (upward concavity), and there is at

\section{Constraints:} most one inflexion point in the right and/or one in the left of the neighbourhood (3)

- Influence area located in a neighbourhood of the target leading line

\section{Examples of possible shape control conditions}

\section{Transversal profiles}

Parameters:

- Curves representing plane profiles

- Positions of profiles with respect to the leading line

Constraints:

- Profiles intersecting the target leading line

- Shape of the profiles: upward concavity in a neighbourhood of each corresponding intersection point with the target leading line, having at most one inflexion point in the right and/or one in the left (3)

- Shape of the profiles: one and only one local and global minimum value inside the upward concavity (3)

- Consistency of the profiles with the geometric continuity conditions $G^{-1}, G^{0}, G^{1}$ or $G^{2}$ on the influence boundary

\section{Transversal curvature laws around the target leading line}

\section{Parameters:}

- Transversal curvature law of the surface around the target leading line ( fixed curvature for transversal profiles intersecting the target leading line at its intersection points)

\section{Constraints:}

- If curvature is given in vectorial form: Frenet normal unit vector at the point of the leading line point concordant with the surface normal unit vector in the same point

\section{Transversal gradient laws around the target leading line}

\section{Parameters:}

- Transversal gradient law of the surface around the target leading line ( fixed tangent for transversal profiles intersecting the target leading line at its intersection points)

\section{Constraints: -}

\section{POCKET}

Derived from: Intrusion type

Name: $\quad$ Pocket feature

Definition: Surface representing an intrusion inside a closed target leading curve, such that in the domain over the influence area every transversal profile has an upward concavity and there is at most an inflexion

Constraints: point in the right side and/or in the left side (3)

- $\quad$ Closed leading line

Examples of possible shape control conditions

\section{Transversal profiles}

Parameters:

- Curves representing plane profiles 
- Positions of profiles with respect to the leading line

Constraints:

- Profiles intersecting the target leading line in two points

- Shape of the profiles: an upward concavity has to exist interiourly to the domain having as extremes the two intersection points with the target leading line, and at most one inflexion point in the right and/or one in the left exist (3)

- Shape of the profiles: one and only one local and global minimum value inside the upward concavity (3)

- Consistency of the profiles with the geometric continuity conditions $G^{-1}, G^{0}, G^{1}$ or $G^{2}$ on the influence boundary

\section{Transversal curvature laws around the target leading line}

\section{Parameters:}

- Transversal curvature law of the surface around the target leading line ( fixed curvature for transversal or parallel profiles intersecting the target leading line at its intersection points)

\section{Constraints:}

- If curvature is given in vectorial form: Frenet normal unit vector at the point of the leading line point concordant with the surface normal unit vector in the same point

\section{Transversal gradient laws around the target leading line}

\section{Parameters:}

- Transversal gradient law of the surface around the target leading line (i.e. fixed tangent at the intersection points of the transversal profiles and the target leading line)

\section{Constraints: -}

\section{STEP-DOWN}

Derived from: Intrusion type

Name: $\quad$ Step down feature

Definition: $\quad$ Surface representing an intrusion lying in a prevailing way from one side of an open target leading line, such that in the domain over the influence area each transversal profile has internally a upward concavity and there exists at most one inflexion point at the right side and at most one at the left side (3)

\section{Constraints:}

- Open leading line

- Influence area greatly asymmetric at the target leading line (i.e. prevailing influence from one side)

\section{Examples of possible shape control conditions}

\section{Transversal profiles}

Parameters:

- Curves representing plane profiles

- Positions of profiles with respect to the leading line

\section{Constraints:}

- Profiles intersecting the target leading line

- Shape of given profiles: an upward concavity exists with at most one inflexion point at most one inflexion point at the right side and at most one at the left side (3)

- Consistency with the geometric continuity conditions $G^{-1}, G^{0}, G^{1}$ or $G^{2}$ on the influence boundary

\section{Transversal curvature laws around the target leading line}

\section{Parameters:}

- Transversal curvature law of the surface around the target leading line (i.e. fixed curvature of the transversal profiles at their intersection points with the target leading line)

\section{Constraints}

- If curvature is given in vectorial form: Frenet normal unit vector at the point of the leading line point concordant with the surface normal unit vector in the same point 


\section{Transversal gradient laws around the target leading line}

\section{Parameters:}

- Transversal gradient law of the surface around the target leading line (i.e. fixed tangent for transversal profiles at their intersection points with the leading line)

Constraints: -

\section{STEP-DOWN-0}

Derived from: Step down feature

Name: $\quad$ Step down 0 feature

Definition: Surface representing a step down feature, where the target leading line is an open line having both extremes not belonging to the leading line, i.e. their position is changed during projection of the leading line onto the target line.

\section{Parameters: -}

\section{Constraints:}

- $\quad$ Non-zero depth vector for both the extremes of the target line with respect to the leading line

\section{STEP-DOWN-1}

Derived from: Step down feature

Name: $\quad$ Step down 1 feature

Definition: Surface representing a step down feature, where the target leading line is an open line having one extreme belonging to the leading line, but not the other one, i.e. one and only one extreme has a

Parameters: position remaining fixed during generation of the target line from the leading line.

\section{Constraints:}

- Zero depth vector of one and only one of the two extremes of the target line with respect to the leading line

- At least $G^{0}$ continuity over the fixed extreme

\section{STEP-DOWN-2}

Derived from: Step down feature

Name: $\quad$ Step down 2 feature

Definition: Surface representing a step down feature, where the target leading line is an open line having both extremes belonging to the leading line, i.e. they both have a position remaining fixed during generation of the target line from the leading line.

\section{Parameters: -}

\section{Constraints:}

- Zero depth vector over both the two extremes of the target line with respect to the leading line

- At least $G^{0}$ continuity over both the fixed extremes

\section{DISPLACEMENT-DOWN}

Derived from: Abstract - Intrusion type

Name: $\quad$ Displacement down feature

Definition: Surface representing an intrusion where a part is obtained by a rigid translation of a part of the primary surface along a fixed direction non tangent to the surface

\section{Parameters:}

- Displacement area

- Direction of the rigid translation

- Depth of the rigid translation

- Geometric continuity conditions $G^{-1}, G^{0}, G^{1}$ or $G^{2}$ over each part of the displacement boundary internal to the influence area

\section{Constraints:}

- Generation law of the target leading line = rigid translation along the given direction at the given depth 
- Generation law to be applied to each point in the displacement area

- Direction of rigid translation different from the direction of any tangent to the primary surface

- Direction of rigid translation discordant with the normal unit vector of the primary surface

\section{POCKET-BY-DISPLACEMENT}

Derived from: Displacement down

Name: $\quad$ Pocket by displacement feature

Definition: $\quad$ Surface representing an intrusion obtained by displacement of a part where the displacement boundary

Parameters:

Constraints:

- Connected closed leading line

- Leading line internal to the influence boundary

- displacement area = region internal to the leading line

- $G^{-1}$ condition not admitted over the displacement boundary

\section{STEP-DOWN-BY-DISPLACEMENT}

Derived from: Displacement down

Name: $\quad$ Step down by displacement feature

Definition: $\quad$ Surface representing an intrusion obtained by displacement where then displacement area crosses the influence boundary

\section{Parameters:}

- Portion of the influence area representing the displacement area $A_{D}$

- Geometric continuity conditions $G^{0}, G^{1}$ or $G^{2}$ over each piece of the displacement boundary internal to the influence area

\section{Constraints}

- Open leading line intersecting the influence boundary in exactly two points (i.e. it splits the influence area $A_{I}$ into two connected parts: $A_{T}$, transition area, and $A_{D}$, displacement area)

- $G^{-1}$ continuity along any part of the displacement boundary contained in the influence boundary and at least a part of the adjacent curves of the influence boundary in the transition area

\section{TYPE EXTRUSION}

Derived from: Abstract - Detail Feature

Name: $\quad$ Extrusion or protrusion type

Definition: Detail shape obtained by a surface deformation having concordant direction with respect to the normal unit vector at the surface for each point belonging to the influence area

Parameters:

- Curves representing the leading basic line

- Relative position of the leading basic line and the influence area as regards the surface

- Projection law for the leading basic line

- Deformation law for the generation of the target leading line

- Shape control conditions

Constraints:

- Non enveloping leading line

- Leading line contained in the influence area

- Law for generating target leading lines such that depth has direction concordant with the normal unit vector at the surface

Examples of possible shape control conditions:

\section{Passage for leading points:}

\section{Parameters:}

- Point co-ordinates in the primary surface 
- $\quad$ Target position for the points (vector depth)

Constraints:

- Points belonging to the influence area

- Vector depth of target points concordant with the normal unit vector at the surface

- For any point belonging to the influence boundary: it must exist a $G^{-1}$ condition set in a neighbourhood of the point

\section{RIB}

Derived from: Extrusion type

Name: $\quad$ Rib feature

Definition: Surface representing an extrusion in a neighbourhood of a target leading line, such that in the domain over the influence area each transversal profile at the line in each point locally has a downward concavity), and there is at most one inflexion point in the right and/or one in the left of the

\section{Constraints:} neighbourhood (3)

- Influence area located in a neighbourhood of the target leading line

Examples of possible shape control conditions:

\section{Transversal profiles}

Parameters:

- Curves representing plane profiles

- Positions of profiles with respect to the leading line

Constraints:

- Profiles intersecting the target leading line

- Shape of the profiles: downward concavity in a neighbourhood of each corresponding intersection point with the target leading line, having at most one inflexion point in the right and/or one in the left (3)

- Shape of the profiles: one and only one local and global maximum value inside the downward concavity (3)

- Consistency of the profiles with the geometric continuity conditions $G^{-1}, G^{0}, G^{1}$ or $G^{2}$ on the influence boundary

\section{Transversal curvature laws around the target leading line}

Parameters:

- Transversal curvature law of the surface around the leading line

\section{Constraints:}

- If curvature is given in vectorial form: Frenet normal unit vector at the point of the leading line point discordant with the surface normal unit vector in the same point

\section{Transversal gradient laws around the leading line}

\section{Parameters:}

- Transversal gradient law of the surface around the target leading line

Constraints: -

\section{BOSS}

Derived from: Extrusion type

Name: $\quad$ Boss feature

Definition: Surface representing an extrusion inside a closed target leading curve, such that in the domain over the influence area every transversal profile has a downward concavity and there is at most an inflexion point in the right side and/or in the left side (3)

\section{Constraints:}

- Closed leading line

Examples of possible shape control conditions:

\section{Transversal profiles}

Parameters: 
- Curves representing plane profiles

- Positions of profiles with respect to the leading line

Constraints:

- Profiles intersecting the leading line in two points

- Shape of the profiles: a downward concavity has to exist interiourly to the domain having as extremes the two intersection points with the target leading line, and at most one inflexion point in the right and/or one in the left exist (3)

- Shape of the profiles: one and only one local and global maximum value inside the upward concavity (3)

- Consistency of the profiles with the geometric continuity conditions $G^{-1}, G^{0}, G^{1}$ or $G^{2}$ on the influence boundary

\section{Transversal curvature laws around the target leading line}

Parameters:

- Transversal curvature law of the surface around the target leading line (i.e. fixed curvature for transversal or parallel profiles at the intersection points with the target leading line)

\section{Constraints:}

- If curvature is given in vectorial form: Frenet normal unit vector at the point of the leading line point discordant with the surface normal unit vector in the same point

\section{Transversal gradient laws around the target leading line}

\section{Parameters:}

- Transversal gradient law of the surface around the target leading line (i.e. fixed tangent for transversal profiles at its intersection points with the leading line)

\section{Constraints: -}

\section{STEP-UP}

Derived from: Extrusion type

Name: $\quad$ Step up feature

Definition: Surface representing an extrusion lying in a prevailing way from one side of an open target leading line, such that in the domain over the influence area each transversal profile has internally a downward concavity and there exists at most one inflexion point at the right side and at most one at

Constraints: the left side (3)

- Open leading line

- Influence area greatly asymmetric at the target leading line (i.e. prevailing influence from one side)

Examples of possible shape control conditions:

\section{Transversal profiles}

Parameters:

- Curves representing plane profiles

- Positions of profiles with respect to the leading line

Constraints:

- Profiles intersecting the target leading line

- Shape of given profiles: a downward concavity exists with at most one inflexion point at the right side and at most one at the left side (3)

- Consistency with the geometric continuity conditions $G^{-1}, G^{0}, G^{1}$ or $G^{2}$ on the influence boundary

\section{Transversal curvature laws around the target leading line}

Parameters:

- Transversal curvature law of the surface around the target leading line (i.e. fixed curvature for transversal profiles at their intersection points with the target leading line)

\section{Constraints:}

- If curvature is given in vectorial form: Frenet normal unit vector at the point of the leading line point discordant with the surface normal unit vector in the same point 


\section{Transversal gradient laws around the target leading line}

Parameters:

- Transversal gradient law of the surface around the target leading line (i.e. fixed tangent for transversal profiles at their intersection points with the leading line)

Constraints: -

\section{STEP-UP-0}

Derived from: Step up feature

Name: $\quad$ Step up 0 feature

Definition: Surface representing a step up feature, where the target leading line is an open line having both extremes not belonging to the leading line, i.e. their position is changed during projection of the leading line onto the target line.

\section{Parameters: -}

\section{Constraints:}

- $\quad$ Non-zero depth vector for both the extremes of the target line with respect to the leading line

\section{STEP-UP-1}

Derived from: Step up feature

Name: $\quad$ Step up 1 feature

Definition: Surface representing a step up feature, where the target leading line is an open line having one extreme belonging to the leading line, but not the other one, i.e. one and only one extreme has a position remaining fixed during generation of the target line from the leading line.

\section{Parameters: -}

\section{Constraints:}

- Zero depth vector of one and only one of the two extremes of the target line with respect to the leading line

- At least $G^{0}$ continuity over the fixed extreme

\section{STEP-UP-2}

Derived from: Step up feature

Name: $\quad$ Step up 2 feature

Definition: Surface representing a step up feature, where the target leading line is an open line having both extremes belonging to the leading line, i.e. they both have a position remaining fixed during

\section{Parameters: -} generation of the target line from the leading line.

\section{Constraints:}

- Zero depth vector over both the two extremes of the target line with respect to the leading line

- At least $G^{0}$ continuity over both the fixed extremes

\section{DISPLACEMENT-UP}

Derived from: Abstract - Extrusion type

Name: $\quad$ Displacement up feature

Definition: Surface representing an extrusion where a part is obtained by a rigid translation of a part of the primary surface along a fixed direction non tangent to the surface

\section{Parameters:}

- Direction of the rigid translation

- Depth of the rigid translation

- Displacement area $A_{D}$

- Geometric continuity conditions $G^{-1}, G^{0}, G^{1}$ or $G^{2}$ over each part of the displacement boundary internal to the influence area

\section{Constraints:}

- Generation law of the target leading line = rigid translation along the given direction at the given depth 
- Generation law to be applied to each point in the displacement area

- Direction of rigid translation different from the direction of any tangent to the primary surface

- Direction of rigid translation concordant with the normal unit vector of the primary surface

\section{BOSS-BY-DISPLACEMENT}

Derived from: Displacement up

Name: $\quad$ Boss by displacement feature

Definition: $\quad$ Surface representing an extrusion obtained by displacement of a part where the displacement boundary is a connected closed line internal to the influence area

\section{Parameters: -}

Constraints:

- Connected closed leading line (=displacement boundary)

- Leading line internal to the influence boundary

- $A_{D}=$ region internal to the leading line

- $G^{-1}$ condition not admitted over the displacement boundary

\section{STEP-UP-BY-DISPLACEMENT}

Derived from: Displacement up

Name: $\quad$ Step up by displacement feature

Definition: $\quad$ Surface representing an extrusion obtained by displacement where then displacement area crosses the

Parameters: influence boundary

- Portion of the influence area representing the displacement area $A_{D}$

- Geometric continuity conditions $G^{0}, G^{1}$ or $G^{2}$ over each piece of the displacement boundary internal to the influence area

\section{Constraints}

- Open leading line intersecting the influence boundary in exactly two points (i.e. it splits the influence area $A_{I}$ into two connected parts: $A_{T}$, transition area, and $A_{D}$, displacement area)

- $G^{-1}$ continuity along any part of the displacement boundary contained in the influence boundary and at least a part of the adjacent curves of the influence boundary in the transition area

\section{TYPE CUT}

Derived from: Abstract - Detail feature

Name: $\quad$ Cut

Definition: Elimination of a part of surface

\section{Parameters:-}

Constraints: -

\section{SHARP-CUT}

\section{Derived from: Abstract - Cut type}

Name: $\quad$ Sharp cut

Definition: Elimination of the influence area from the primary surface

Parameters: -

Constraints: -

- Transition conditions along the influence boundary $=G^{-1}$ conditions

- $\quad$ Deformation law $=$ surface trim along the influence boundary

\section{SHARP-HOLLOW}

Derived from: Sharp cut type

Name: $\quad$ Sharp hollow cut


Definition: Elimination of a part of the surface at its boundary by maintaining the local simple connection

Parameters: -

Constraints:

- Influence area crossing the surface boundary

- Simply connected influence area

- Conservation of the surface local simple connection degree

\section{SHARP-HOLE}

Derived from: Sharp cut type

Name: Sharp hole cut

Definition: Elimination of an internal part of the surface

Parameters: -

Constraints:

- Influence area non crossing the surface boundary

- Simply connected influence area

\section{SHARP-GAP}

Derived from: Sharp cut type

Name: Sharp gap cut

Definition: Elimination of a part of the surface producing two totally disconnected surfaces

Parameters: -

- Curve representing the basic leading line

- Relative position of the basic leading line and the influence area as regards the surface**

- Projection law for obtaining the leading line from the basic leading line

Constraints:

- Two disconnected surfaces adjacent to the cut to be obtained

- Leading line contained in the influence boundary

\section{SMOOTH-CUT}

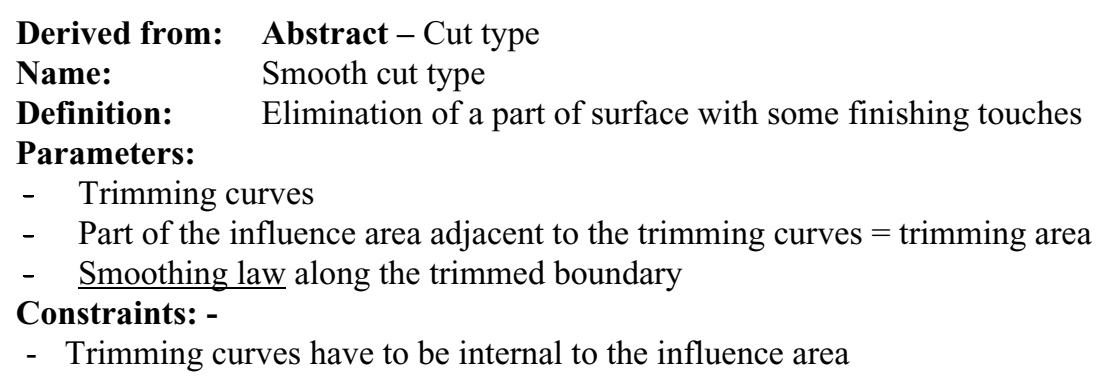

\section{SMOOTH-HOLLOW}

Derived from: Smooth cut type

Name: Smooth hollow cut

Definition: Elimination of a part of the surface at its boundary with some finishing touches by maintaining the local simple connection

Parameters:

- Specific smoothing conditions

Constraints:

- Influence area crossing the surface boundary

- Simply connected influence area

- Trimmed area crossing the surface boundary

- Simply connected trimmed area

- Conservation of the surface local simple connection 


\section{SMOOTH-HOLE}

Derived from: Smooth cut type

Name: $\quad$ Smooth hole cut

Definition: Elimination of an internal part of the surface with some finishing touches

Parameters:

- Specific smoothing conditions

Constraints:

- Influence area non crossing the surface boundary

- Simply connected influence area

- Simply connected trimmed area

\section{SMOOTH-GAP}

Derived from: Smooth cut type

Name: Smooth gap cut

Definition: Elimination of a part of the surface producing two totally disconnected surfaces with some finishing touches

Parameters:

- Curve representing the leading basic line

- Relative position of the leading basic line and the influence area as regards the surface

- Projection law for obtaining the leading line from the leading basic line

- Specific smoothing conditions

Constraints:

- Two disconnected surfaces adjacent to the cut to be obtained

- Leading line contained in the influence boundary 


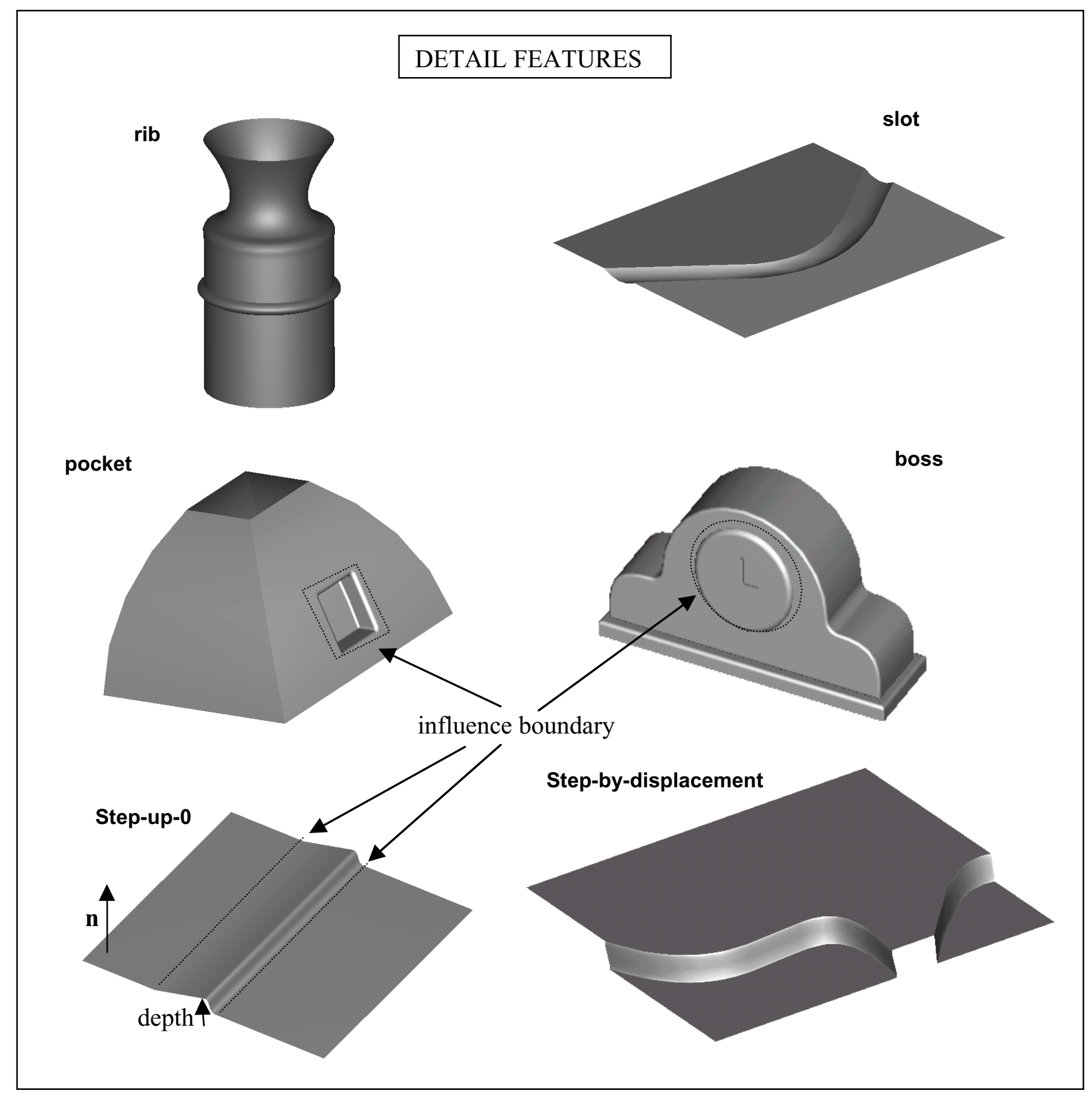

Fig. 34: Examples of detail features 


\section{Acknowledgements}

This work is supported by the European Commission under the Brite-Euram Project $\mathrm{N}^{\circ}$ BE96-3579. The authors are grateful to the FIORES partners, prof. Bianca Falcidieno for the helpful discussion on the topic.

\section{Bibliography}

[1] J.J. Shah, M. Mantyla, 'Parametric and feature-based CAD/CAM', Wiley-Interscience

[2] G. Farin, 'Curves and surfaces for computer aided geometric design - A practical guide', Academic Press, Inc.

[3] L. Piegl, W. Tiller, 'The NURBS book', Springer

[4] T. De Martino, F. Giannini, B. Falcidieno, 'Features and Styling', FIORES technical report, July 1997

[5] T. De Martino, F. Giannini, 'Mathematical problems related to feature definition /instantiation', FIORES Technical Report, July 1997

[6] T. De Martino, F. Giannini,, 'Feature-based description of the test-cases', FIORES technical report, November 1997

[7] T. De Martino, F. Giannini, 'Integration of the free form feature concept into the final FIORES prototype', FIORES Technical Report, February 1998

[8] M. Mata Dumenjo, P. Brunet, 'Dictionary of terms', FIORES technical report, November 1998

[9] M. Meirana, 'Metodi matematici per la caratterizzazione di superfici nella progettazione estetica', Tesi di laurea, Università di Genova, 1997

[10] T. De Martino, M. Meirana, 'Caratterizzazione estetica di superfici free-form', Technical report, IMA n. 12/97, CNR Genova, 1997

[11] J.C. Cavendish, 'A procedural feature-based approach for designing functional surfaces',

[12] J.C. Cavendish, S.P. Marin, 'Feature-based surface design and machining', IEEE Computer Graphics and Applications, 1992

[13] G. Wyvill, D McRobie, C. Haig, 'Free form modelling with history',

[14] T. Varay, R.R. Martin, J. Cox, 'Reverse engineering of geometric models - an introduction', Computer Aided Design, Vol.29, 255-268, 1997

[15] M. Hoffmann, R. Joan-Arinyo,'On user-defined features', Computer Aided Design, Vol.30, 321-332, 1998

[16] C. Sacchi, 'Feature based shaping of parametric surfaces through variable distance offsetting', KAEMaRT Technical Report KTR-1/95

[17] G. Durand, A. Leutier, A. Massabo, 'On aesthetic preserving algorithm for accuracy incompatible modelers data exchange', Technical Matra DataVision, IDMNE 96

[18] P. Bosinco, G. Durand, J. Goussard, A: Lieutier, A. Massabo, 'Complex shape modifications', Technical report Matra DataVision, IDMNE 98 